\title{
RefPlanets: Search for reflected light from extrasolar planets with SPHERE/ZIMPOL *
}

\author{
S. Hunziker ${ }^{1}$, H. M. Schmid ${ }^{1}$, D. Mouillet ${ }^{3,4}$, J. Milli ${ }^{5}$, A. Zurlo ${ }^{17,18,15}$, P. Delorme ${ }^{3}$, L. Abe ${ }^{12}$, H. Avenhaus ${ }^{13,1}$, \\ A. Baruffolo ${ }^{14}$, A. Bazzon ${ }^{1}$, A. Boccaletti ${ }^{10}$, P. Baudoz ${ }^{10}$, J. L. Beuzit ${ }^{15}$, M. Carbillet ${ }^{12}$, G. Chauvin ${ }^{3,16}$, R. Claudi ${ }^{14}$, \\ A. Costille ${ }^{15}$, J.-B. Daban ${ }^{12}$, S. Desidera ${ }^{14}$, K. Dohlen ${ }^{15}$, C. Dominik ${ }^{9}$, M. Downing ${ }^{19}$, N. Engler ${ }^{1}$, M. Feldt ${ }^{13}$, \\ T. Fusco ${ }^{15,20}$, C. Ginski ${ }^{11}$, D. Gisler ${ }^{7,8}$, J. H. Girard ${ }^{24,3}$, R. Gratton ${ }^{14}$, Th. Henning ${ }^{13}$, N. Hubin ${ }^{19}$, M. Kasper ${ }^{19}$, \\ C. U. Keller ${ }^{11}$, M. Langlois ${ }^{21,15}$, E. Lagadec ${ }^{12}$, P. Martinez ${ }^{12}$, A. L. Maire ${ }^{13,23}$, F. Menard ${ }^{3,4}$, M. R. Meyer ${ }^{25}$, \\ A. Pavlov ${ }^{13}$, J. Pragt ${ }^{2}$, P. Puget ${ }^{3}$, S. P. Quanz ${ }^{1}$, E. Rickman ${ }^{22}$, R. Roelfsema ${ }^{2}$, B. Salasnich ${ }^{14}$, J.-F. Sauvage ${ }^{15,20}$, \\ R. Siebenmorgen ${ }^{19}$, E. Sissa ${ }^{14}$, F. Snik ${ }^{11}$, M. Suarez ${ }^{19}$, J. Szulágyi ${ }^{6}$, Ch. Thalmann ${ }^{1}$, M. Turatto ${ }^{14}$, S. Udry ${ }^{22}$, \\ R. G. van Holstein ${ }^{11}$, A. Vigan ${ }^{15}$, and F. Wildi ${ }^{22}$
}

(Affiliations can be found after the references)

Received 5 September 2019 / Accepted 24 November 2019

\begin{abstract}
Aims. RefPlanets is a guaranteed time observation programme that uses the Zurich IMaging POLarimeter (ZIMPOL) of SpectroPolarimetric High-contrast Exoplanet REsearch instrument at the Very Large Telescope to perform a blind search for exoplanets in wavelengths from 600 to $900 \mathrm{~nm}$. The goals of this study are the characterisation of the unprecedented high polarimetic contrast and polarimetric precision capabilities of ZIMPOL for bright targets, the search for polarised reflected light around some of the closest bright stars to the Sun, and potentially the direct detection of an evolved cold exoplanet for the first time.

Methods. For our observations of $\alpha$ Cen A and B, Sirius A, Altair, $\epsilon$ Eri and $\tau$ Ceti we used the polarimetric differential imaging (PDI) mode of ZIMPOL which removes the speckle noise down to the photon noise limit for angular separations $\gtrsim 0.6^{\prime \prime}$. We describe some of the instrumental effects that dominate the noise for smaller separations and explain how to remove these additional noise effects in post-processing. We then combine PDI with angular differential imaging as a final layer of post-processing to further improve the contrast limits of our data at these separations.

Results. For good observing conditions we achieve polarimetric contrast limits of 15.0-16.3 mag at the effective inner working angle of $\sim 0.13^{\prime \prime}, 16.3-18.3 \mathrm{mag}$ at $0.5^{\prime \prime}$, and $18.8-20.4 \mathrm{mag}$ at $1.5^{\prime \prime}$. The contrast limits closer in $\left(\lesssim 0.6^{\prime \prime}\right)$ display a significant dependence on observing conditions, while in the photon-noise-dominated regime $\left(\gtrsim 0.6^{\prime \prime}\right)$ the limits mainly depend on the brightness of the star and the total integration time. We compare our results with contrast limits from other surveys and review the exoplanet detection limits obtained with different detection methods. For all our targets we achieve unprecedented contrast limits. Despite the high polarimetric contrasts we are not able to find any additional companions or extended polarised light sources in the data obtained so far.
\end{abstract}

Key words. instrumentation: high angular resolution - methods: data analysis - methods: observational - techniques: polarimetric techniques: image processing - planets and satellites: detection

\section{Introduction}

High-contrast imaging is a key technique for the search and classification of extrasolar planets, which is one of the primary goals in modern astronomy. However, the technical requirements are very challenging and up to now only about a dozen young, giant planets have been directly imaged (e.g., Macintosh et al. 2015; Bowler 2016; Schmidt et al. 2016; Chauvin et al. 2017; Keppler et al. 2018). Young, self-contracting giant planets are hot, with temperatures of $T \approx 1000-2000 \mathrm{~K}$ (e.g. Baraffe et al. 2003; Spiegel \& Burrows 2012). Therefore, they are bright in the nearinfrared (NIR) and the required contrast $C=F_{\mathrm{pl}} / F_{\text {star }} \approx 10^{-5 \pm 1}$ is within reach of modern extreme adaptive optics (AO) systems, like the Spectro-Polarimetric High-contrast Exoplanet REsearch

\footnotetext{
* Based on observations made with ESO Telescopes at the La Silla Paranal Observatory under programme IDs: 095.C-0312(B), 096.C0326(A), 097.C-0524(A), 097.C-0524(B), 098.C-0197(A), 099.C0127(A), 099.C-0127(B), 0102.C-0435(A).
}

instrument (SPHERE; Beuzit et al. 2008) at the Very Large Telescope (VLT), the Gemini Planet Imager (GPI; Macintosh et al. 2014), the Natural Guide Star (NGS) AO system (van Dam et al. 2004) at Keck or the Subaru Coronagraphic Extreme Adaptive Optics (SCExAO; Jovanovic et al. 2015). Unfortunately, young stars with planets are rare in the solar neighbourhood. Furthermore, for the young stars in the nearest star forming regions at $d \approx 150 \mathrm{pc}$ the expected angular separations of planets tend to already be quite small and therefore these objects are difficult to detect in observations.

Most old planets, including all habitable planets, are cold and therefore produce only scattered light in the visual to NIR $(<2 \mu \mathrm{m})$ wavelength range (Sudarsky et al. 2003). Lightscattering by the atmosphere of a planet produces a polarisation signal that can be distinguished from the unpolarised light of the much brighter central star (Seager et al. 2000; Stam et al. 2004; Buenzli \& Schmid 2009). The contrast of this reflected light from extrasolar planets with respect to the brightness of their host stars is very challenging $\left(C \lesssim 10^{-7}\right)$, but polarimetric 
differential imaging (PDI) has been shown to be a very effective technique to reveal faint reflected light signals. For these reasons the SPHERE "planet finder" instrument includes the Zurich IMaging POLarimeter (ZIMPOL; Schmid et al. 2018) which was designed for the search of light from reflecting planets in the visual wavelength range using innovative polarimetric techniques (Schmid et al. 2006a; Thalmann et al. 2008).

We investigate in this paper the achievable contrast of SPHERE/ZIMPOL for a first series of deep observations of promising targets obtained within the RefPlanets project, which is a part of the guaranteed time observation (GTO) program of the SPHERE consortium. One important goal of this study is a better understanding of the limitations of this instrument in order to optimise the SPHERE/ZIMPOL observing strategy for high-contrast targets, and possibly to conceive upgrades for this instrument or improve concepts for future instruments, for example for the Extremely Large Telescope (ELT; Kasper et al. 2010; Keller et al. 2010). Pushing the limits of high-contrast imaging polarimetry should be useful for the future investigation of many types of planets around the nearest stars, including Earth twins.

In Sect. 2 we describe the expected polarisation signal from reflecting planets and the search strategy using SPHERE/ ZIMPOL. The GTO observations are presented in Sect. 3, and Sect. 4 discusses our standard data reduction procedures for ZIMPOL polarimetry. Section 5 provides the description of the angular differential imaging method that we applied to our data and the metric for the assessment of the point-source contrast. Section 6 shows our detailed search results for $\alpha$ Cen A. Section 7 discusses the physical meaning of the contrast limits in more detail and Sect. 8 presents our conclusions. In Appendices A and B we present the advanced data-reduction steps necessary to reach the best possible polarimetric contrast limits with ZIMPOL and in Appendix $C$ we present and discuss the detection limits for all other targets of our survey.

\section{Reflected light from exoplanets}

\subsection{The polarisation of the reflected light}

The expected polarisation signal from reflecting planets has been described with simple models (Seager et al. 2000), with detailed calculations for Jupiter and Earth-like planets for example (Stam et al. 2004; Stam 2008), or for a parameter grid of planets with Rayleigh scattering atmospheres (Buenzli \& Schmid 2009; Bailey et al. 2018). The intensity and polarised intensity phase functions depending on the orbital phase angle $\phi$ and the planetstar-observer scattering angle $\alpha$ for one such model is illustrated in Fig. 1. These models are guided by polarimetric observations of Solar System objects for which the typical fractional polarisation is quite high, namely $p(\alpha)>10 \%$, for visible wavelengths and scattering angles in the range $\alpha \approx 60^{\circ}-120^{\circ}$ (e.g. Schmid et al. 2006a).

Observations of individual objects have shown that for Rayleigh scattering atmospheres like Uranus and Neptune (Schmid et al. 2006b) the fractional polarisation can be substantially higher than this value $\left(p\left(90^{\circ}\right)>20 \%\right)$. For mostly haze-scattering atmospheres as found on Titan (Tomasko \& Smith 1982; Bazzon et al. 2014) or in the polar regions of Jupiter (Smith \& Tomasko 1984; Schmid et al. 2011; McLean et al. 2017) the fractional polarisation can even reach values of up to $p\left(90^{\circ}\right) \approx 50 \%$. On the other hand, the Mie scattering process in the clouds that dominate the atmospheres of Venus, Saturn, and the equatorial regions of Jupiter produces a lower polarisation in the visual wavelengths of $<10 \%$ (Smith \& Tomasko 1984; Hansen \& Hovenier 1974). For larger objects without any significant atmosphere like the Moon, Mercury, Mars, and other rocky bodies (e.g. Dollfus 1985) the polarisation of the reflected light is somewhere in the range $p\left(90^{\circ}\right) \approx 5-20 \%$. Finally, for the polarisation of Earth, Bazzon et al. (2013) determined fractional polarisations of about $19 \%$ in $V$-band and $13 \%$ in $R$-band mainly caused by Rayleigh scattering in the atmosphere.

For Rayleigh scattering, haze scattering, and the reflection from solid planet surfaces, the resulting polarisation for $\alpha \approx$ $30^{\circ}-150^{\circ}$ is perpendicular to the scattering plane, as illustrated in Fig. 1a. This means that for extrasolar planets the polarisation is usually positive in the direction perpendicular to the line connecting star and planet as projected onto the sky. However, the polarisation can be negative for the reflection from clouds as observed for Venus (Hansen \& Hovenier 1974), or for reflections with small scattering angles $\left(\alpha \lesssim 25^{\circ}\right)$ on rocky or icy surfaces (Dollfus 1985).

\subsection{The signal from extrasolar planets}

The signal of a reflecting planet depends on the surface properties, which define the reflectivity $I(\alpha)$ and the fractional polarisation $p(\alpha)$ of the planet, as well as the planet size and its separation from the central star. The reflectivity and polarisation depend on the scattering angle $\alpha$ given by the orbital phase $\phi$ and orbit inclination $i$ as sketched in Fig. 1a. We set the phase $\phi=0$ in conjunction, when the planet illumination as seen by the observer is maximal. For circular orbits the dependence is

$\alpha=\arccos (\sin i \cdot \cos \phi)$,

and the scattering angle varies between a minimum and maximum value $\alpha_{\min }$ and $\alpha_{\max }$ as indicated in Fig. 1a. For edge-on orbits $\left(i=90^{\circ}\right)$, Eq. (1) simplifies to $\alpha=|\phi|$ for $\phi=-180^{\circ}$ to $180^{\circ}$, and for pole-on systems $\left(i=0^{\circ}\right)$ we see one single scattering angle of $\alpha=90^{\circ}$ during the whole orbit. Small and large scattering angles of $\alpha \lesssim 30^{\circ}$ and $\alpha \gtrsim 150^{\circ}$ are only observable for strongly inclined orbits $i>60^{\circ}$, but at the corresponding phase angles, planets are typically faint in polarised flux (see Fig. 1b). In addition, the angular separation is small and therefore a successful detection will be particularly difficult (e.g. Schworer \& Tuthill 2015).

For Rayleigh-like scattering the fractional polarisation $p(\alpha)$ is highest around $\alpha \approx 90^{\circ}$ while the reflectivity $I(\alpha)$ increases for $\alpha \rightarrow 0^{\circ}$. Therefore, the maximum polarised intensity $p(\alpha) I(\alpha)$ is expected for a scattering angle of $\alpha \approx 60^{\circ}$. The full dependence of the normalised intensity $I(\phi)$ and polarised intensity $p(\phi) I(\phi)$ as a function of orbital phase for a Rayleigh-scattering planet is illustrated in Fig. 1b. The figure shows simulated phase functions for planets on circular orbits with inclinations of $i=0^{\circ}, 30^{\circ}, 60^{\circ}$, and $90^{\circ}$.

The model in Fig. 1b was selected from the model grid of Rayleigh-scattering atmospheres derived in Buenzli \& Schmid (2009). We use it as a reference case for the reflected intensity and polarisation of a planetary atmosphere. The model is similar to Uranus and Neptune which are quite favourable cases for a polarimetric search for planets. A giant planet might have a thinner Rayleigh scattering layer $\tau_{\mathrm{sc}}<1$ on top of a cloud layer, resulting in a lower polarisation fraction (see Buenzli \& Schmid 2009). This is because the reflection from a cloud layer produces significantly less polarisation than the reflection from a thick $\left(\tau_{\mathrm{sc}} \gtrsim 1\right)$ Rayleigh scattering layer. The model shown in Fig. 1b has an optical depth of $\tau_{\mathrm{sc}}=2$ for the Rayleigh scattering layer, with a single scattering albedo of $\omega=0.95$ above a cloud layer approximated by a Lambertian surface with an albedo of $A_{S}=1$. For quadrature phase, this model yields $\alpha=90^{\circ}$, a reflectivity 
(a)

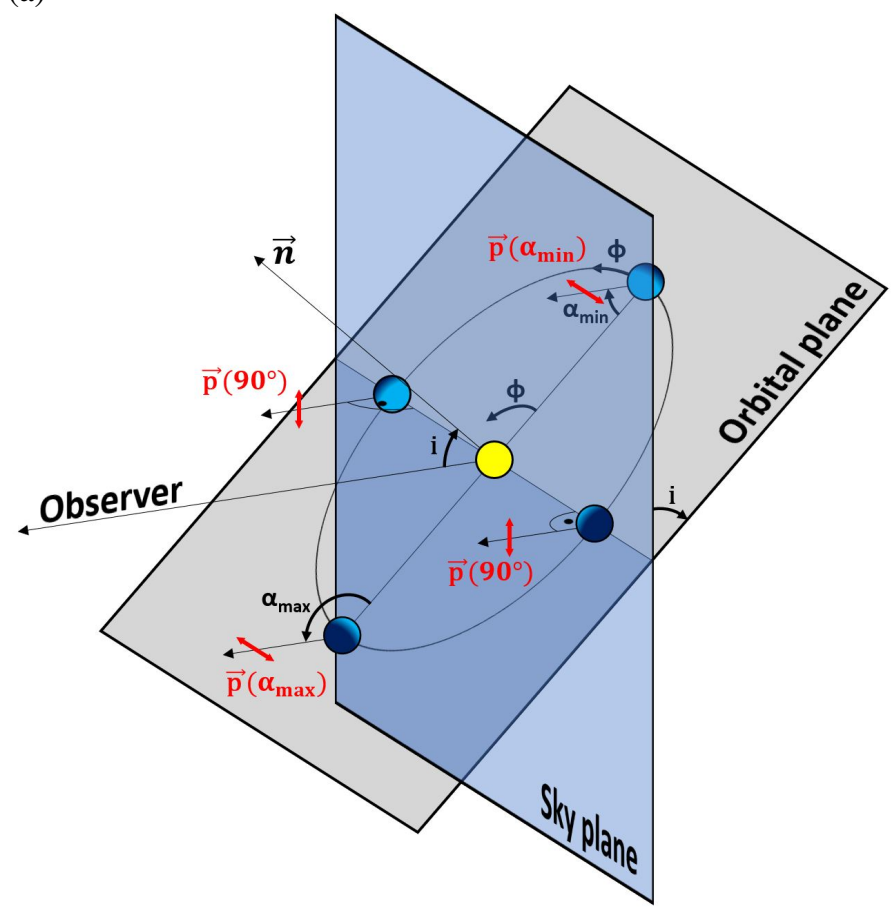

(b)
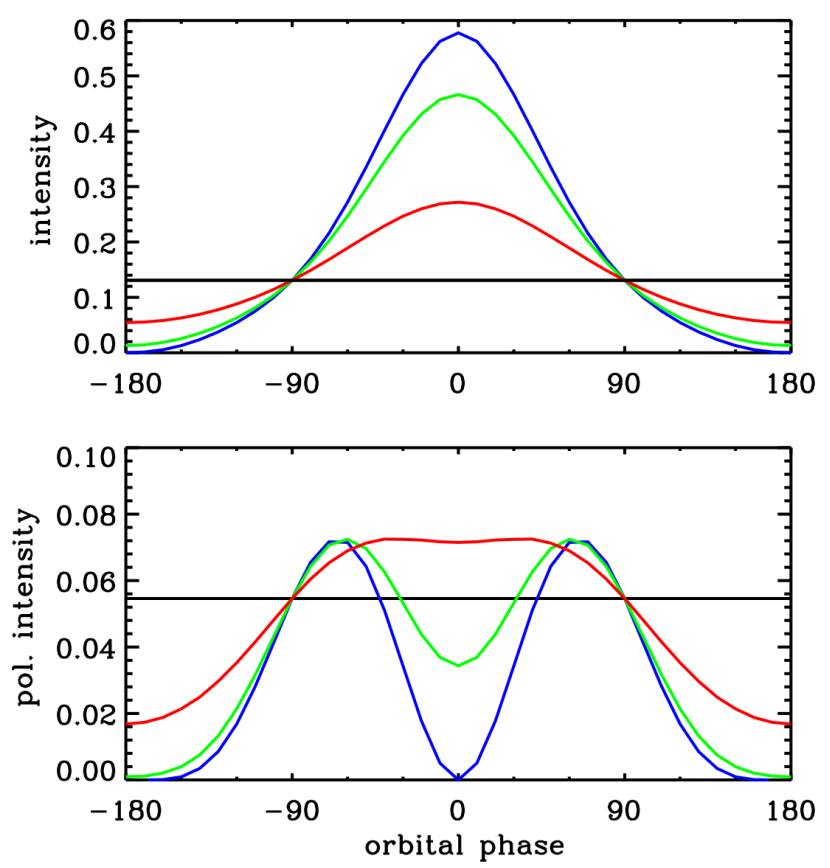

Fig. 1. Panel a: diagram showing the essential planes and angles needed to characterise the reflected light intensity: the orbital phase of the planet $\phi$, the inclination of the orbital plane with respect to the sky plane $i$ and the scattering angle $\alpha$. The scattering angle $\alpha$ is measured along the scattering plane and our definition of the direction of a positive polarisation $p(\alpha)$ is perpendicular to this plane. In special cases the polarisation of the reflected light could be negative. This would correspond to a direction of $p(\alpha)$ perpendicular to the red arrows. Panel $b$ : normalised intensity and polarised intensity of the reflected light as a function of the orbital phase $\phi$, calculated with the reference planet atmosphere model used in this paper: a Rayleigh scattering atmosphere from Buenzli \& Schmid (2009) with an optical depth of $\tau_{\mathrm{sc}}=2$, a single scattering albedo of $\omega=0.90$, and a ground surface (= cloud) albedo of $A_{S}=1$. The different colours show the phase functions of planets on circular orbits seen at four different inclinations: $0^{\circ}$ (black), $30^{\circ}$ (red), $60^{\circ}$ (green), and $90^{\circ}$ (blue).

of $I\left(90^{\circ}\right)=0.131$, and a corresponding polarised signal amplitude of $p\left(90^{\circ}\right) \cdot I\left(90^{\circ}\right)=0.055$. The parameter $p\left(90^{\circ}\right)$ provides a good way to characterise the polarisation of an extrasolar planet because planets at all inclinations will pass through this phase at least twice. In this phase, the fractional polarisation is expected to be close to the maximum and the apparent separation from the star is maximised for planets on circular orbits (see Figs. 1b and 2).

The polarisation $p(\alpha)$ refers to the amplitude of the polarisation but our raw data consists of independent measurements of the Stokes $Q$ and $U$ parameters. Since we can assume that the reflected light from a planet is polarised along the axis perpendicular to the connecting line between star and planet, we use the transformation into polar coordinates from Schmid et al. (2006b) to derive $Q_{\phi}$ and $U_{\phi}$. In the dominating single scattering scenario, the tangential polarisation $Q_{\phi}$ should contain all the polarised intensity of the reflected light, while $U_{\phi}$ should be zero everywhere. Because of this relationship we use $Q_{\phi}$ to denote the polarised intensity throughout this work.

The key parameter for the polarimetric search of reflecting extrasolar planets is the polarisation contrast $C_{\mathrm{pol}}$, which is the polarised flux $Q_{\phi}$ from the planet relative to the total flux from the central star:

$C_{\text {pol }}(\alpha)=p(\alpha) \cdot C_{\text {flux }}(\alpha)=p(\alpha) \cdot I(\alpha) \frac{R_{\mathrm{p}}^{2}}{d_{\mathrm{p}}^{2}}$,

where $R_{\mathrm{p}}$ is the radius of the planet, $d_{\mathrm{p}}$ the physical separation between planet and star, and $I(\alpha)$ and $p(\alpha)$ are the reflectivity and fractional scattering polarisation for a given scattering angle, respectively. In this notation, the reflectivity $I(\alpha=0)$ is equivalent to the geometric albedo $A_{\mathrm{g}}$ of a planet. The ratio $R_{\mathrm{p}}^{2} / d_{\mathrm{p}}^{2}$ for a Jupiter-sized planet with radius $R_{\mathrm{J}}$ at a separation $d_{\mathrm{p}}=1 \mathrm{AU}$ is $R_{\mathrm{J}}^{2} / \mathrm{AU}^{2}=2.3 \times 10^{-7}$ and the total polarisation contrast of a planet with our reference model with $p\left(90^{\circ}\right) \cdot I\left(90^{\circ}\right)=0.055$ would be of order $C_{\text {pol }} \approx 10^{-8}$. A Neptune-sized planet would have to be located at about $0.5 \mathrm{AU}$ to produce the same polarisation contrast. With increasing physical separation $d_{\mathrm{p}}$ the contrast decreases rapidly with $1 / d_{\mathrm{p}}^{2}$ (see Eq. (2)). With increasing distance to the star, the angular separation $\rho$ of a planet at a constant $d_{\mathrm{p}}$ also decreases, moving it closer to the star where high contrasts cannot be maintained. The combination of both effects limits the sample of possible targets for a search of reflected light to the most nearby stars. In addition, the sample is limited to only the brightest stars because photon noise increases like $1 / \sqrt{F}$ with the lower photon flux $F$ of stars that are fainter in the visible wavelengths.

\subsection{Targets for the search of extrasolar planets}

The detection space for our SPHERE/ZIMPOL high-contrast observations starts at about $\rho \approx 0.1^{\prime \prime}$, and the current polarimetric contrast limits after post-processing are of the order $10^{-7}$ for $\rho<0.5^{\prime \prime}$ and $10^{-8}$ for $\rho>0.5^{\prime \prime}$. Therefore, only the nearest stars within about $5 \mathrm{pc}$ can have a sufficiently bright reflecting planet with $R_{\mathrm{p}} \approx R_{\mathrm{J}}$ and a contrast of $C_{\mathrm{pol}} \gtrsim 10^{-8}$ with a sufficiently large angular separation $\rho>0.1^{\prime \prime}$ for a successful detection. Based on these criteria, some of the best stellar systems for the search of a Jupiter-sized planet in reflected light 


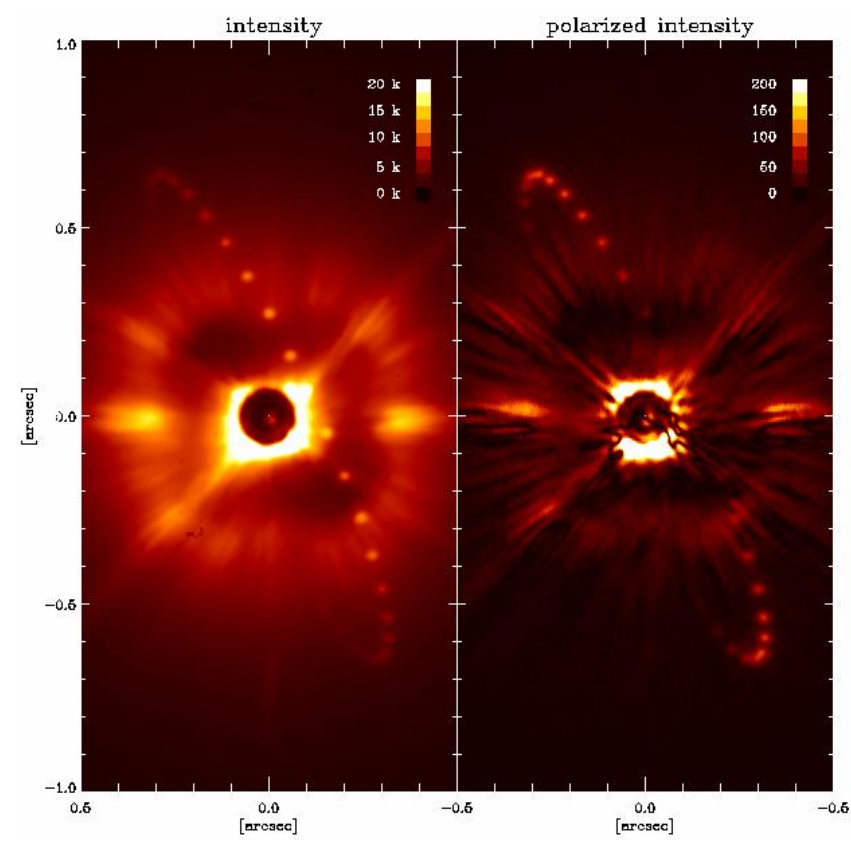

Fig. 2. Apparent positions of a model planet on a circular $80^{\circ}$ inclined orbit with $r=1$ AU around $\alpha$ Cen A in a typical coronagraphic intensity (left) and polarisation frame (right) at ten-day intervals. The brightness of the planet signal with respect to $\alpha$ Cen A is exaggerated by a factor of $10^{4}$ for the intensity and $10^{3}$ for the polarisation. The relative brightness of the point for different phases is according to the model presented in Fig. 1.

with SPHERE/ZIMPOL are $\alpha$ Cen A and B, Sirius A, $\epsilon$ Eri, $\tau$ Cet, Altair, and a few others as determined by Thalmann et al. (2008).

No extrasolar planet is known to exist around these highpriority stars which would fulfil the above detection limit criteria. There is strong evidence from radial velocity and astrometric studies for the presence of a giant planet in $\epsilon$ Eri (e.g. Hatzes et al. 2000; Mawet et al. 2019), but the derived separation is $3 \mathrm{AU}$ and therefore the expected signal is at the level of only $C_{\text {pol }} \approx 10^{-9}$. For $\tau$ Cet, the presence of planets has been proposed based on radial velocity data (Feng et al. 2017), but no planet is expected to produce a contrast of $C_{\text {pol }} \gtrsim 10^{-9}$. The radial velocity constraints for the A-stars Sirius A and Altair are very loose because their spectra are not well suited to sensitive radial velocity searches, and undetected giant planets at $1 \mathrm{AU}$ may be present. The radial velocity limits for planets are very stringent for $\alpha$ Cen B (Zhao et al. 2018), but less well constrained for $\alpha$ Cen A (Zhao et al. 2018). However, the simple calculation of the reflected light contrast does not consider the possibility that a planet could be exceptionally bright due to certain reasons; such as for example an extensive ring system surrounding the planet (e.g. Arnold \& Schneider 2004). In light of the absence of obvious targets, we decided to carry out an exploratory blind search for "unexpectedly" bright companions, additionally aiming to investigate the detection limits of SPHERE/ZIMPOL and to define the best observing strategies for possible future searches.

For such a survey, one needs to consider that planets around the nearest stars are moving quickly through our field of view (FOV). This is illustrated in Fig. 2, which simulates the orbit of a planet with a circular orbit with a separation of $1 \mathrm{AU}$ around $\alpha$ Cen A on top of single coronagraphic intensity or polarimetric frames. The individual points are the orbital positions of this model planet separated by 10 days. The relative brightnesses of the points are calculated for an orbit inclination of $i=80^{\circ}$ coplanar with the $\alpha$ Cen binary (Kervella et al. 2016) and using the same Rayleigh scattering atmosphere model as in Fig. 1, but with the brightness upscaled by a factor of $10^{4}$ for the intensity and $10^{3}$ for the polarisation in order to make the dots visible on top of a single coronagraphic observation. Furthermore, the angular motion depends on the orbital parameters and the distance of the systems and our example $\alpha$ Cen A system would show, for a planet, the fastest angular orbital motion for a given orbital separation because of its proximity.

Without going into details, the $\alpha$ Cen A example in Fig. 2 already illustrates that planets on inclined orbits have phases with large separation when they are relatively bright and easy to detect, and phases where they are close to the star and faint and challenging to detect. Therefore, a blind search provides only planet detection limits valid for that observing date. One should also notice that data taken during different nights cannot simply be coadded for the search of extrasolar planets due to the expected short orbital periods. Instead, a tool like K-Stacker (Nowak et al. 2018) is necessary, which combines the results from multiple epochs while considering the orbital motion of a planet.

\section{Observations}

\subsection{The SPHERE/ZIMPOL instrument}

The polarimetric survey for extrasolar planets was carried out with the SPHERE "Planet Finder" instrument (Beuzit et al. 2008, 2019) on the VLT Unit Telescope 3 (UT3) at ESO. SPHERE is an extreme AO system with a fast tip-tilt mirror and a fast high-order deformable mirror with $41 \times 41$ actuators and a Shack-Hartman wave-front sensor (e.g. Fusco et al. 2006). The system includes an image de-rotator, atmospheric dispersion correctors, calibration components, and the IRDIS (Dohlen et al. 2008), IFS (Claudi et al. 2008), and ZIMPOL focal plane instruments for high-contrast imaging.

This program was carried out with ZIMPOL which was specifically designed for the polarimetric search of reflected light from extrasolar planets around the nearest bright stars in the spectral range 500-900 $\mathrm{nm}$. The SPHERE/ZIMPOL system is described in detail in Schmid et al. (2018) and here we highlight some of the important properties for high-contrast imaging of reflected light from planets:

- The polarimetric mode is based on a fast modulationdemodulation technique which reaches a polarimetric sensitivity ${ }^{1}$ of $\Delta p<10^{-4}$ (Schmid et al. 2018) in the light halo of a bright star. This is possible because the used modulation frequency of $968 \mathrm{~Hz}$ is faster than the seeing variations and therefore the speckle noise suppression for PDI is particularly good as long as the coherence time $\tau_{0}$ is greater than about $2 \mathrm{~ms}$. This condition was usually satisfied during the RefPlanets observations (see Table 1).

- ZIMPOL polarimetry can be combined with coronagraphy for the suppression of the diffraction-limited PSF peak of the bright star for a sensitive search of faint point-sources in the light halo of a bright star.

- ZIMPOL has a small pixel scale of 3.6 mas pix $^{-1}$, a detector mode with a high pixel gain of $10.5 \mathrm{e}^{-} \mathrm{ADU}^{-1}$, and a full well capacity of $640 \mathrm{ke}^{-} \mathrm{pix}^{-1}$. This allows searches for very faint polarised signals in coronagraphic images of very bright stars,

Degree of suppression of the light by the polarimetry. 
S. Hunziker et al.: RefPlanets: search for reflected light from extrasolar planets with SPHERE/ZIMPOL

Table 1. Summary of all RefPlanets observations completed until the end of 2018.

\begin{tabular}{|c|c|c|c|c|c|c|c|c|c|c|c|}
\hline \multirow[t]{2}{*}{ Date (UT) } & \multirow[t]{2}{*}{ Object } & \multirow{2}{*}{$\begin{array}{l}m_{\mathrm{R}} \\
(\mathrm{mag})\end{array}$} & \multicolumn{2}{|c|}{ Filters } & \multirow{2}{*}{$\begin{array}{l}\text { DIT } \\
(\mathrm{sec})\end{array}$} & \multirow{2}{*}{$\begin{array}{l}\text { \# of } \\
\text { pol. } \\
\text { cycles }\end{array}$} & \multirow[t]{2}{*}{$t_{\exp }^{(a)}$} & \multirow{2}{*}{$\begin{array}{l}\text { Seeing } \\
\left({ }^{\prime \prime}\right)\end{array}$} & \multirow{2}{*}{$\begin{array}{l}\tau_{0} \\
(\mathrm{~ms})\end{array}$} & \multirow[t]{2}{*}{ Air mass } & \multirow{2}{*}{$\begin{array}{l}\text { Field } \\
\text { rotation } \\
\left(^{\circ}\right)\end{array}$} \\
\hline & & & cam1 & cam2 & & & & & & & \\
\hline /01 & $A$ & -0.5 & I & R & 1.2 & 66 & 2 & 0 & .5 & 17 & 90.4 \\
\hline 2015/05/02 & $\alpha$ Cen B & 1.0 & $\mathrm{VBB}$ & R_PRIM & 1.2 & 90 & $3 \mathrm{~h} 36 \mathrm{~min}$ & $0.6-1.1$ & $1.5-2.2$ & $1.24-1.46$ & 97.1 \\
\hline $2016 / 02 / 17$ & Sirius A & -1.5 & N_I & N_I & 1.2 & 34 & $1 \mathrm{~h} 21.6 \mathrm{~min}$ & $0.7-2.0$ & & $1.01-1.13$ & 101.1 \\
\hline 2016/02/20 & Sirius A & -1.5 & N_I & N_I & 1.2 & 73 & 2 h $55.2 \mathrm{~min}$ & $1.0-2.0$ & $1.8-3.5$ & 1.01-1.39 & 113.2 \\
\hline 2016/04/18 & $\alpha$ Cen A & -0.5 & N_R & N_R & 1.2 & 40 & $1 \mathrm{~h} 36 \mathrm{~min}$ & $1.1-1.5$ & $1.8-2.4$ & $1.26-1.48$ & 46.7 \\
\hline $2016 / 04 / 21$ & $\alpha$ Cen A & -0.5 & N_R & N_R & 1.2 & 80 & $3 \mathrm{~h} 12 \mathrm{~min}$ & $0.7-1.7$ & $2.0-4.0$ & $1.24-1.71$ & 107.9 \\
\hline $2016 / 06 / 22$ & $\alpha$ Cen B & 1.0 & $\mathrm{VB} B$ & $\mathrm{VB} B$ & 1.1 & 74 & $.8 \mathrm{~min}$ & $0.3-0.8$ & 5.0 & $1.24-1.60$ & 106.6 \\
\hline $2016 / 07 / 21$ & Altair & 0.6 & R_PRIM & R_PRIM & 1.2 & 63 & $.2 \mathrm{~min}$ & $0.4-0.8$ & 7.0 & $1.20-1.44$ & 67.2 \\
\hline $2016 / 07 / 22$ & Altair & 0.6 & R_PRIM & R_PRIM & 1.2 & 30 & $1 \mathrm{~h} 12 \mathrm{~min}$ & $0.4-0.7$ & $3.0-5.0$ & $1.20-1.32$ & 27.6 \\
\hline $2016 / 10 / 10$ & $\epsilon$ Eri & 3.0 & $\mathrm{VBB}$ & VBB & 3.0 & 42 & $2 \mathrm{~h} 48 \mathrm{~min}$ & $0.5-0.8$ & $4.5-6.7$ & $1.05-1.37$ & $0^{(b)}$ \\
\hline $2016 / 10 / 11$ & $\epsilon$ Eri & 3.0 & VBB & VBB & 3.0 & 48 & $3 \mathrm{~h} 12 \mathrm{~min}$ & $0.5-0.8$ & $3.3-6.5$ & 1.04-1.18 & $\mathbf{0}^{(b)}$ \\
\hline 2016/10/12 & $\epsilon$ Eri & 3.0 & VBB & VBB & 5.0 & 15 & $1 \mathrm{~h} 40 \mathrm{~min}$ & $1.0-1.8$ & $1.8-2.1$ & $1.04-1.21$ & $0^{(b)}$ \\
\hline $2017 / 04 / 30$ & $\alpha \operatorname{Cen} A$ & -0.5 & N_R & N_R & 1.2 & 84 & $3 \mathrm{~h} 21.6 \mathrm{~min}$ & $0.5-0.7$ & $3.0-4.4$ & $1.24-2.21$ & 121.5 \\
\hline $2017 / 05 / 01$ & $\alpha$ Cen A & -0.5 & N_R & N_R & 1.2 & 39 & $1 \mathrm{~h} 33.6 \mathrm{~min}$ & $0.8-1.3$ & $2.0-2.5$ & $1.37-1.84$ & 47.8 \\
\hline 2017/06/19 & $\alpha$ Cen B & 1.0 & VBB & VBB & 1.1 & 141 & 3 h $26.8 \mathrm{~min}$ & $0.3-1.0$ & 4.5-9.5 & $1.24-1.52$ & 115.6 \\
\hline 2018/10/14 & $\tau$ Ceti & 2.9 & R_PRIM & R_PRIM & 14 & 30 & $2 \mathrm{~h} 48 \mathrm{~min}$ & 0.4-0.7 & 5.0-10 & 1.01-1.12 & 130.5 \\
\hline $2018 / 10 / 15$ & $\tau$ Ceti & 2.9 & R_PRIM & R_PRIM & 14 & 24 & $2 \mathrm{~h} 14.4 \mathrm{~min}$ & $0.6-1.6$ & $2.0-4.0$ & $1.01-1.12$ & 112.1 \\
\hline 2018/10/16 & $\tau$ Ceti & 2.9 & R_PRIM & R_PRIM & 14 & 32 & $2 \mathrm{~h} 59 \mathrm{~min}$ & $0.6-1.0$ & $2.5-3.7$ & $1.01-1.28$ & 104.9 \\
\hline 2018/10/19 & $\tau$ Ceti & 2.9 & R_PRIM & R_PRIM & 14 & 29 & $2 \mathrm{~h} 42.4 \mathrm{~min}$ & $0.6-1.4$ & $2.6-5.3$ & $1.01-1.60$ & 107.5 \\
\hline
\end{tabular}

Notes. For each observation we also list observing conditions (seeing in arc seconds and coherence time $\tau_{0}$ in ms) and the total field rotation (relevant for the efficiency of angular differential imaging). The datasets with the deepest limits for each target are marked in bold font. ${ }^{(a)}$ The total exposure time per camera. ${ }^{(b)} \epsilon$ Eri was observed in the field-stabilised ZIMPOL P2-polarimetry mode.

$m_{R}<4^{m}$, with broadband filters by "simply" pushing the photon noise limit thanks to the photon collecting power of the VLT telescope.

The combination of high-contrast imaging using $\mathrm{AO}$ and coronagraphy provides a raw contrast at a level of $10^{-4}-10^{-5}$ for point-sources, while polarimetry in combination with angular differential imaging (ADI) yields a further contrast improvement for polarimetric differential imaging of about $10^{-3}$, so that a total contrast of $C_{\text {pol }} \approx 10^{-8}$ is reachable with sufficiently long integrations.

\subsection{Observations}

Table 1 lists all the observations that we have carried out so far for the RefPlanets GTO program. We observed six of the most favourable targets in the solar neighbourhood identified by Thalmann et al. (2008) as ideal targets for the search of planets in reflected light.

All data were taken with the fast modulation polarimetry mode, which is the mode of choice for high-flux applications. The first observations in 2015 were made with different filters in cameras 1 and 2 of ZIMPOL. However, it was noticed that some disturbing polarimetric residuals can be corrected if the simultaneous camera 1 and camera 2 frames are taken with the same filter passband, because the residuals have opposite signs and compensate when camera 1 and camera 2 frames taken with the same filter are combined. Indeed, the contrast also improves with the combination of data from both cameras because of the lower photon noise limit. From 2016 onwards we took for each hour of coronagraphic observations one or two short polarimetric cycles with the star offset from the focal plane mask for the calibration of the flux, the point-spread function (PSF), and the polarimetric beam shift (Schmid et al. 2018). These PSFs were taken with neutral density (ND) filters to avoid detector saturation.
The main criterion for the filter selection is a high photon throughput. Filters with broader passbands provide more photons and stars with $m_{R}>1^{m}$ were observed usually in the VBB filter $\left(\lambda_{c, \mathrm{VBB}}=735 \mathrm{~nm}, \Delta \lambda_{\mathrm{VBB}}=291 \mathrm{~nm}\right)$. For Sirius A, $\alpha$ Cen A, and Altair we used filters with smaller band widths to avoid detector saturation with the minimum detector integration time of $1.1 \mathrm{~s}$ available for ZIMPOL, namely, the R_PRIM $\left(\lambda_{c, \text { R_PRIM }}=626 \mathrm{~nm}, \Delta \lambda_{\text {R_PRIM }}=149 \mathrm{~nm}\right)$, N_R $\left(\lambda_{c, \mathrm{~N} \_\mathrm{R}}=646 \mathrm{~nm}, \Delta \lambda_{\mathrm{N}_{-} \mathrm{R}}=57 \mathrm{~nm}\right)$, and N_I $\left(\lambda_{c, \mathrm{~N}_{-} \mathrm{I}}=817 \mathrm{~nm}\right.$, $\left.\Delta \lambda_{N_{-} I}=81 \mathrm{~nm}\right)$ filters. Only for $\tau$ Ceti did we deviate from this strategy and chose the R_PRIM filter instead of the VBB filter. This is because we noticed that certain disturbing wavelengthdependent instrumental effects (instrumental polarisation, beam shift) are easier to correct during the data reduction for narrower passbands.

Almost all objects were observed with SPHERE/ZIMPOL in $\mathrm{P} 1$-mode, in which the image de-rotator is fixed. In this mode the sky rotates as a function of the telescope parallactic angle and altitude allowing for ADI (Marois et al. 2008) in combination with PDI because most of the strong aberrations - mainly caused by the deformable mirror (DM) - are fixed with respect to the detector. The P1-mode stabilises the instrument polarisation after the HWP2-switch, but does not stabilise the telescope pupil, which still rotates with the telescope altitude. Therefore, speckles related to the telescope pupil cannot be suppressed with ADI. We observed only $\epsilon$ Eri in the field-stabilised polarimetric P2-mode to make use of the improved capability of the instrument to detect weak extended scattering polarisation from circumstellar dust which could be detectable with our FOV of 3.6" × 3.6" (e.g. Backman et al. 2009; Greaves et al. 2014).

For all observations we used the medium-sized classical Lyot coronagraph CLC-MT-WF with a dark focal plane mask spot deposited on a plate with a radius corresponding to 77.5 mas (Schmid et al. 2018). However, the effective inner working angle 
(IWA) of the reduced data is generally larger and depends on the star centring accuracy and stability. The spot in this coronagraphic mask has a transparency of about $0.1 \%$ (Schmid et al. 2018) and during good conditions and with good centring the star is visible behind the coronagraph so that an accurate centring of the frames is possible.

Our usual observing strategy for deep coronagraphic observations consists of one-hour blocks, each with about five to ten polarimetric cycles. Each cycle consists of observations with all four half-wave plate orientations $\left(Q^{+}, Q^{-}, U^{+}, U^{-}\right)$. Between these blocks we took short non-coronagraphic cycles with a neutral density filter, by offsetting the star from the coronagraphic mask to acquire samples of the unsaturated PSF for image quality assessments, flux calibrations, and the measurement of the beam-shift effect.

\section{Basic data reduction}

The data reduction is mainly carried out with the Interactive Data Language (IDL) based ZIMPOL software pipeline developed at ETH Zurich. Basic data preprocessing, reduction, and calibration steps are essentially identical to the ESO Data Reduction and Handling (DRH) software package developed for SPHERE (Pavlov et al. 2008). The basic steps are described briefly in this section and more technical information is available in Schmid et al. (2012, 2018). In addition, we describe in the Appendix the more advanced sz-pipeline routines and additional data-reduction procedures required especially for high-contrast imaging and polarimetry.

The fast modulation and on-chip demodulation imaging polarimetry of ZIMPOL produces raw frames where the simultaneous $I_{\perp}$ and $I_{\|}$polarisation signals are registered on alternating rows of the CCD detectors. Briefly, the ZIMPOL raw polarisation signal $Q^{Z}$ is the difference between the "even-row" $I_{\perp}$ and the "odd-row" $I_{\|}$subframes, $Q^{Z}=I_{\perp}-I_{\|}$. The raw intensity signal is derived from adding the two subframes: $I^{Z}=I_{\perp}+I_{\|}$.

Just like for any other CCD detector data, the basic datareduction steps include image extraction, frame flips for the correct image orientation, a first bias subtraction based on the pre- and overscan pixel level, bias frame subtraction for fixed pattern noise removal, and flat-fielding. Special steps for the ZIMPOL system are the differential polarimetric combination of the subframes, taking into account the alternating modulation phases for the CCD pixel charge trap correction (Gisler et al. 2004; Schmid et al. 2012), and calibrating the polarimetric efficiency $\epsilon_{\mathrm{pol}}$ or modulation-demodulation efficiency. The polarimetric combination of the frames of a polarimetric cycle $Q^{+}, Q^{-}, U^{+}, U^{-}$taken with the four half-wave plate orientations is again done in a standard way. For non-fieldstabilised observations, the data combination must also consider the image rotation. As a basic data product of one polarimetric cycle one obtains four frames, $I_{Q}, Q, I_{U}$, and $U$, which can be combined with the frames from many other cycles for higher signal-to-noise ratio $(\mathrm{S} / \mathrm{N})$ results.

The basic PDI data-reduction steps listed above are not sufficient for reaching the very high polarimetric contrast required for the search of reflecting planets. Especially at smaller separations of $\lesssim 0.6^{\prime \prime}$ the noise is still dominated by residuals of order $10^{-6}$ in terms of contrast compared to the brightness of the star (see Fig. 3). This is why we additionally apply more advanced calibration steps described in Appendices A and B. These steps include frame transfer smearing correction, telescope polarisation correction, and correction of the differential polarimetric beam shift.

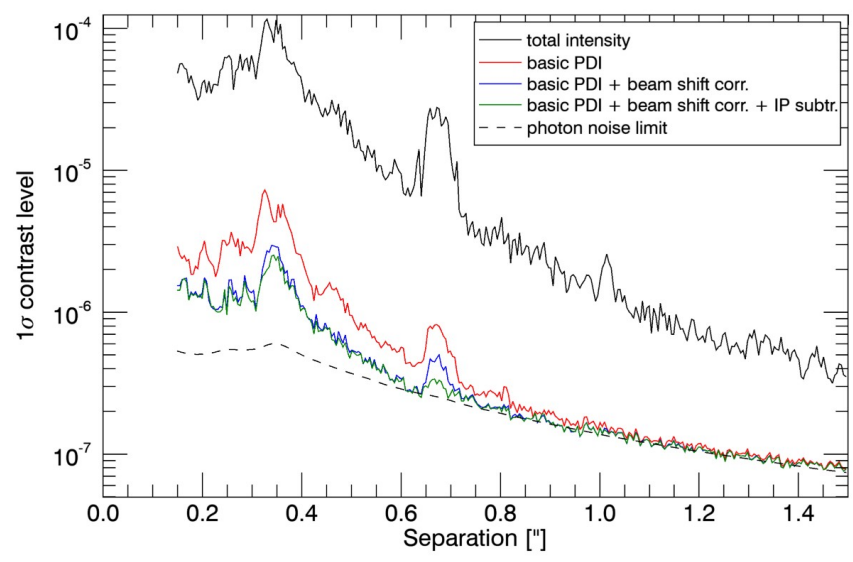

Fig. 3. $1 \sigma$ radial contrast levels for the data shown after the different major data-reduction steps - basic PDI by ZIMPOL, correction of the polarimetric beam shift, and subtraction of instrument polarisation (IP) - for the polarised intensity $Q$ and for the corresponding intensity $I$ of one single combined zero-phase and $\pi$-phase $(2 \times 1.2 \mathrm{~s})$ exposure of $\alpha$ Cen A in the N_R filter.

\section{Post-processing and the determination of the contrast limits}

There is still a landscape of residual noise visible after the basic data reduction, beam shift and frame transfer smearing correction, and the subtraction of the residual instrument polarisation. This can be seen for example in the bottom panel of Fig. A.3. We show this quantitatively with $1 \sigma$ noise levels for a series of short $2.4 \mathrm{~s}$ exposures measured after the different reduction steps in Fig. 3. After the full data reduction, the residual noise at small separations $<0.6^{\prime \prime}$ still dominates the photon noise by a factor of between approximately two and five in this particular example. For larger separations $>0.6^{\prime \prime}$ the residual noise is close to the photon noise limit. In an effort to further reduce the noise at small separations, we used a principle component analysis(PCA)-based ADI algorithm (Amara \& Quanz 2012) to model the fixed and slowly varying residual noise features.

\subsection{Principle-component-analysis-based angular differential imaging}

The top row in Fig. 4 shows the coronagraphic total intensity and polarised intensity data of $\alpha$ Cen A from June 2017 after de-rotating and combining all frames. The intensity exhibits a PSF speckle halo with a strong radial gradient over two orders of magnitude. The differential polarisation shows arc-like patterns in the de-rotated and combined image which originate from the de-rotated fixed residual noise pattern. Angular differential imaging can be used to efficiently model and subtract such largescale patterns before de-rotating and combining the images. The PCA-ADI approach was used successfully by van Holstein et al. (2017) to improve the contrast limits of SPHERE/IRDIS polarimetry data.

We used a customised version of the core code from the PynPoint pipeline (Amara \& Quanz 2012; Stolker et al. 2019) for the ADI process. The complete speckle-subtraction process was applied to the stacks of $Q^{+}, Q^{-}, U^{+}, U^{-}$and intensity frames separately after preprocessing and centering the frames. For the polarised intensity frames we applied PCA in an annulus around the star from $0.1^{\prime \prime}$ to $1^{\prime \prime}$ in order to cover the speckle-dominated region. For the total intensity frames we increased the outer radius to $1.8^{\prime \prime}$ since the whole FOV is dominated by speckles and 

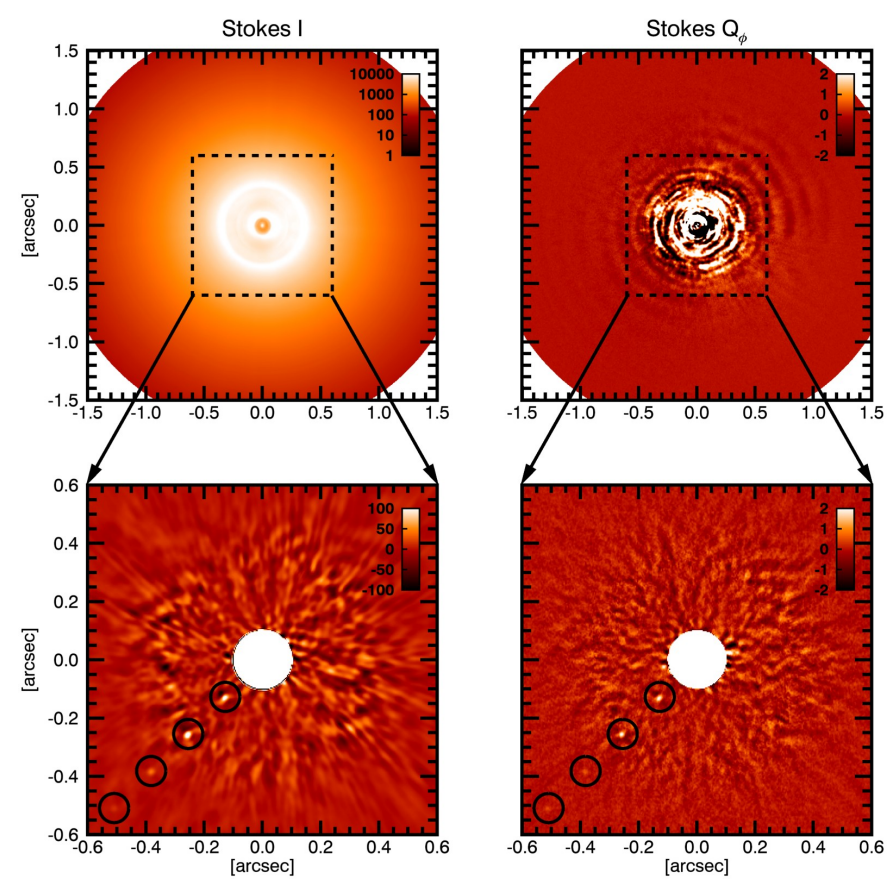

Fig. 4. Total intensity (Stokes $I$ ) and polarised intensity (Stokes $Q_{\phi}$ ) for the complete dataset of $\alpha$ Cen A in the N_R filter. The frames in the bottom row show a closer look at the speckle-dominated region closer to the star after injecting artificial point-sources (black circles) and applying PCA-ADI with 20 PCs.

other fixed pattern noise. We used a fixed number of 20 principle components (PCs), or $10 \mathrm{PCs}$ in the case of the $\tau$ Ceti polarimetry, to model and subtract the residual noise patterns because this seemed to be the number that produced deep contrast limits at most separations. In the bottom row of Fig. 4 we show an example of the result after removing 20 PCs from the intensity and polarised intensity frames. In both cases the contrast improved significantly. Typical contrast limit improvements for PCA-ADI were between a factor of five and ten for the total intensity and up to a factor of three for the polarised intensity. In Fig. 4, the resulting images after PCA-ADI also contain a number of artificial planets with $S / N \approx 5$, they were introduced for estimating the contrast limits after PCA-ADI.

For the de-rotation and combination of the frames, we applied the noise-weighted algorithm as described in Bottom et al. (2017). This algorithm is simple to implement in a direct imaging data-reduction pipeline and often significantly improved the $\mathrm{S} / \mathrm{R}$ of the artificial planets, with typical S/N gains of about $8 \%$ and a maximum gain up to $26 \%$ for our $\alpha$ Cen A test dataset.

\subsection{Polarimetric point-source contrast}

The contrast limits after the PCA-ADI step were calculated using artificial point sources arranged in a spiral pattern around the star which we tried to recover with $S / N=5$. We determined the $\mathrm{S} / \mathrm{N}$ using the methods derived in Mawet et al. (2014), including the correction for small sample statistics. The artificial planet PSF was simulated with a non-coronagraphic PSF from one of the beam-shift measurements, upscaled with the mean value of the transmission curve for the neutral density filter that was used to avoid saturation. We visually selected the non-coronagraphic PSF that best fits the shape of the coronagraphic PSF of the combined intensity image at separations $>0.3^{\prime \prime}$. This ensures that we do not severely over- or underestimate the aperture flux that a point source would have in our data, and therefore guarantees

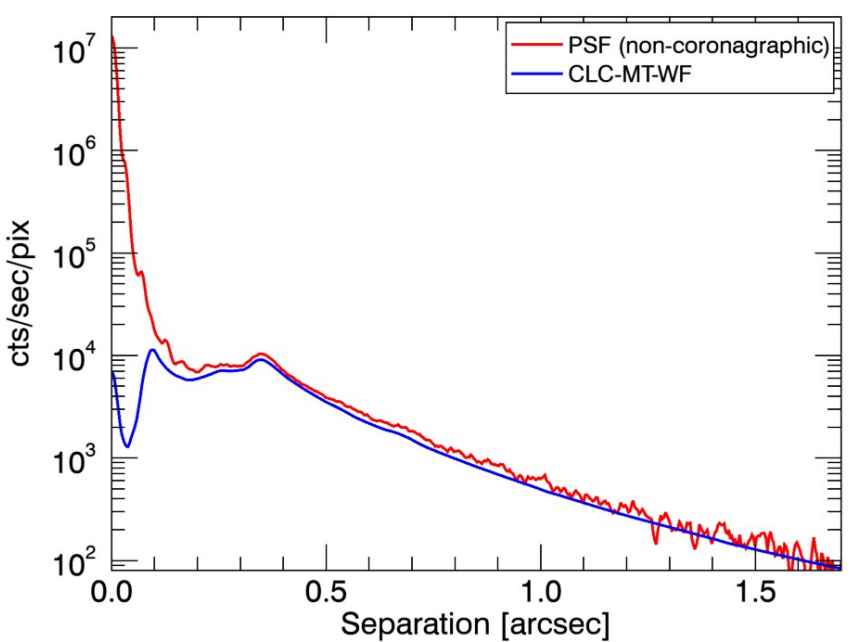

Fig. 5. Coronagraphic PSF with the CLC-MT-WF coronagraph (blue) compared to the non-coronagraphic PSF of $\alpha$ Cen A in the N_R filter (red). The coronagraphic PSF was upscaled by a factor of $\sim 8 \times 10^{3}$ to account for the use of a neutral density filter during the measurement.

accurate contrast limit estimations. For the $\alpha$ Cen A data, we show the radial profiles of both PSFs in Fig. 5, normalised to the number of counts on the detector per second and pixel.

The aperture radius $r_{\text {ap }}$ used for the contrast estimation and $\mathrm{S} / \mathrm{N}$ calculation was optimised for high $\mathrm{S} / \mathrm{N}$ under the assumption that the sought-after point source is weak compared to the PSF of the central star and read-out noise is negligible. With increasing $r_{\mathrm{ap}}$, the number of counts from a faint source increases; however, a larger aperture also has an increased background noise $\sigma_{\text {bck }} \propto r_{\text {ap }}$. We derived an optimised $r_{\text {ap }} \approx \lambda / D$, corresponding to about 4-6 pixels (14-22 mas) depending on the observed wavelength. The flux in each aperture was background subtracted individually using the mean value of the pixels in a two-pixel-wide concentric annulus around the aperture, because the point-source contrast should not be affected by residual, nonaxisymmetric, large-scale structures in the image (e.g. stray light from $\alpha$ Cen $\mathrm{A}$ in the observations of $\alpha$ Cen B).

We also calculated raw contrast curves for both Stokes $I$ and $Q_{\phi}$ without PCA-ADI to investigate how the other advanced data-reduction steps improve the contrast limits at different separations. The calculated raw contrast curves do not require the insertion of fake signals and are independent of the field rotation. Therefore, the raw contrast is more suitable for assessing the quality of small subsets of the data or even single exposures.

For the raw contrast we also used methods derived in Mawet et al. (2014) to calculate the noise at different separations to the star and turn this into the signal aperture flux required for a detection. The detection threshold was set to a constant false positive fraction (FPF) corresponding to the FPF of an $\mathrm{N} \sigma$ detection with Gaussian distributed noise. The required aperture flux was then turned into a contrast limit estimation by dividing it through the aperture flux of the unsaturated stellar PSF.

In order to apply the signal detection method described in Mawet et al. (2014), the underlying distribution of noise aperture fluxes has to be approximately Gaussian. We applied a Shapiro-Wilk test and found that this condition is satisfied for all separations.

\section{Results for $\alpha$ Cen A}

We present a detailed analysis of the results from the deepest observations of $\alpha$ Cen A. We derive contrast limits and analyse 
(a)

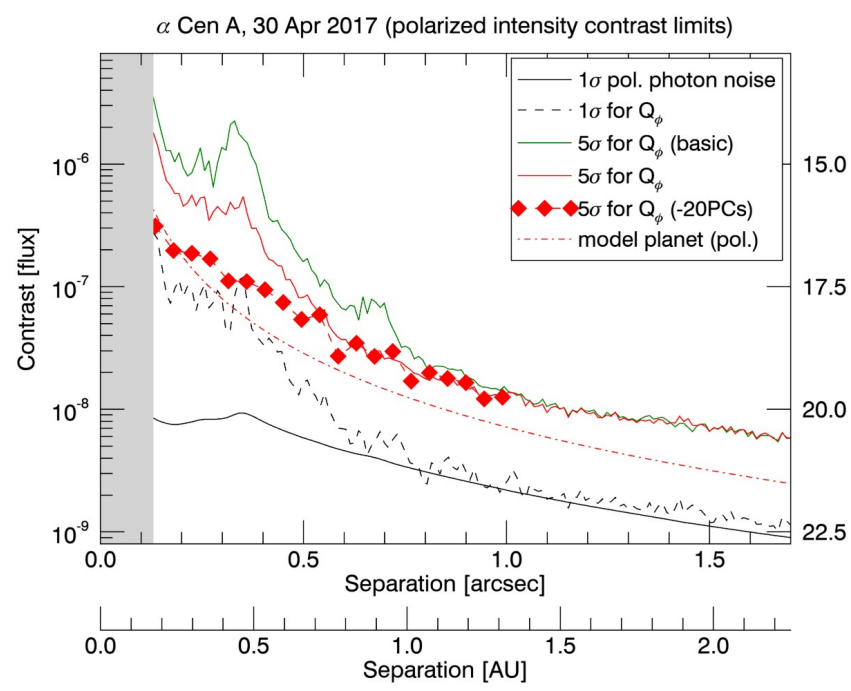

(b)

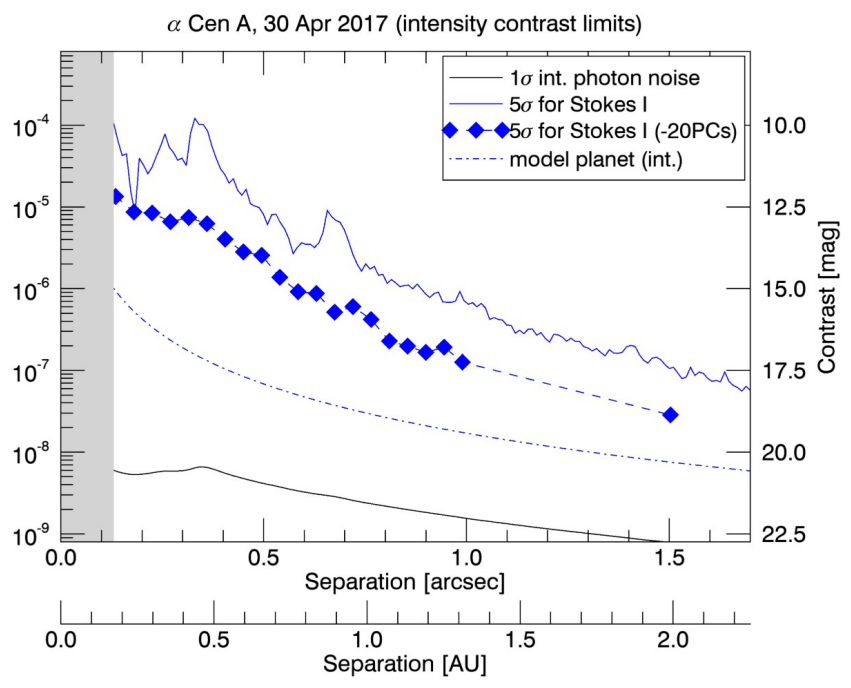

Fig. 6. Radial contrast limits as a function of separation for the deepest ZIMPOL high-contrast dataset of $\alpha$ Cen A in the N_R filter. Panel $a$ : $1 \sigma$ and $5 \sigma$ limits for the polarised intensity as well as the $1 \sigma$ photon noise limit for the polarised intensity. The basic data reduction (green line) was done without beam-shift correction and residual instrument polarisation subtraction; the complete reduction includes both steps. The diamond symbols show the improvement of the contrast limits after applying PCA-ADI. Panel $b$ : $5 \sigma$ limits for the intensity as well as the $1 \sigma$ photon noise limit for the intensity. For both contrast limits - polarised intensity and intensity - we also show the corresponding contrast of our reference model planet.

the properties of the noise at different separations and for different total detector integration times (DITs). This detailed analysis shows the outstanding performance of ZIMPOL in terms of speckle suppression in PDI mode. In addition to that, we present and discuss the best results for all other targets of our survey in Appendix C.

\subsection{Total intensity and polarised intensity}

The deepest observations of $\alpha$ Cen A were carried out in the N_R filter and in P1 polarimetry mode during a single half-night with good observing conditions (see Table 1). For the full data reduction we used the best 84 out of 88 polarimetric cycles with a total $t_{\text {exp }}$ of $201.6 \mathrm{~min}$ for each camera. This is our longest exposure time with a narrowband filter. We combined the results of both cameras to improve the photon noise limit by an additional factor of around $\sqrt{2}$. The resulting de-rotated and combined images are shown in Fig. 4. Just as described in Sect. 2.2, we transformed the polarised intensity frames $Q, U$ into the $Q_{\phi}, U_{\phi}$ basis. We expect a positive $Q_{\phi}$ and no $U_{\phi}$ signal from the reflected light of a companion. The bottom panels of Fig. 4 show the inner, speckledominated region after inserting four artificial point sources in the lower left corner and subsequently removing 20 PCs modes. The final image for $Q_{\phi}$ is clean and shows no disturbing residuals except for a few very close to the coronagraph. However, the total intensity shows some strong disturbing features that are extended in the radial direction. These features are residuals from the diffraction pattern of the rotating telescope spiders. The residuals are unpolarised and are therefore mostly cancelled in the $Q_{\phi}$ result.

\subsection{Contrast curve}

Figure $6 a$ shows the $1 \sigma$ and $5 \sigma$ contrast limits for polarised intensity $Q_{\phi}$ together with the $1 \sigma$ photon noise limit. The contrast is limited by speckle noise when the photon noise is lower than the measured $1 \sigma$ point-source contrast, which is the case for separations $\lesssim 0.6^{\prime \prime}$, corresponding to $\lesssim 1 \mathrm{AU}$ for $\alpha$ Cen A.
The solid green line shows the $5 \sigma$ contrast limits after applying the basic data-reduction steps without beam-shift correction and residual instrument polarisation subtraction; the solid red line includes both additional corrections. The symbols show the corresponding contrast improvements after additional PCA speckle subtraction.

The additional corrections - including PCA-ADI - improve the contrast limits mostly in the speckle-noise-dominated region close to the star at separations $\lesssim 0.5^{\prime \prime}$. The contrast can be improved to about two to five times the fundamental limit due to photon noise for these separations. For separations $\gtrsim 0.5^{\prime \prime}$ the improvement for the polarised intensity is zero but the limits are already close to the photon noise and ADI could only make it worse. This is why we have chosen to apply ADI in combination with PDI only in an annulus instead of applying it to the whole frame.

The solid blue line in Fig. $6 \mathrm{~b}$ is the contrast limit for the total intensity. The corresponding photon noise limit for the intensity is a factor of $\sqrt{2}$ lower than the photon noise limit for the polarisation shown in Fig. 6 a because only $50 \%$ of the photons contribute to the polarised signal $Q_{\phi}$. For the total intensity contrast we also applied PCA-ADI and calculated the resulting contrast limits inside $1^{\prime \prime}$ and at $1.5^{\prime \prime}$. The results show that speckle noise dominates at all separations. The PCA-ADI procedure can be used to improve the limits but they still exceed the photon noise limit by factors of about 100-1000. However, the detection limits for the total intensity could be further improved with the ZIMPOL pupil-stabilised imaging mode without polarimetry. This should produce better contrast limits for the same exposure time.

\subsection{Companion size limit}

The detection limits can be turned into size upper limits for a planet with some assumptions about its reflective properties and orbital phase. We again adopt the reference model from Sect. 2.2 with $Q\left(90^{\circ}\right)=p\left(90^{\circ}\right) \cdot I\left(90^{\circ}\right)=0.055$ and use the contrast curve from Sect. 6.2 to calculate the upper radius limits for 


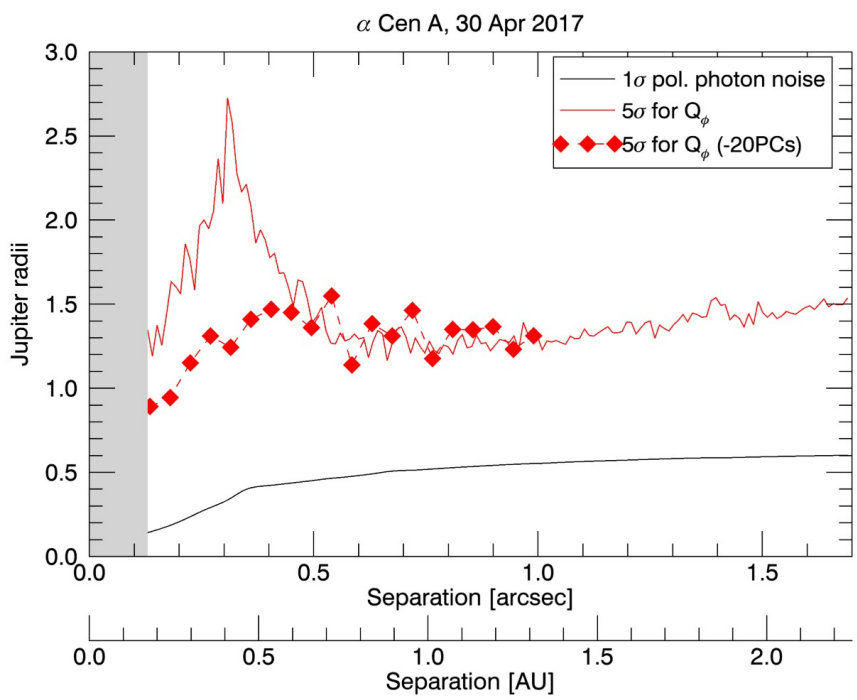

Fig. 7. Polarised intensity contrast limits for $\alpha$ Cen A turned into the minimum size of a planet that could be observed at each apparent separation. We assume that planets are at maximum apparent separation $\left(\alpha=90^{\circ}\right)$ and we adopt our reference model from Sect. 2.2 for the reflective properties of the light.

a companion that would still be detectable with polarimetry. The $5 \sigma$ limits shown in Fig. 7 result in sizes smaller than $1 R_{\mathrm{J}}$ for small separations $\sim 0.2 \mathrm{AU}\left(0.15^{\prime \prime}\right)$ and stay in the range $1-1.5 R_{\mathrm{J}}$ within the whole FOV. The sensitivity improves considerably towards smaller separations (short-period planets) because the brightness of the planet scales with $d_{\mathrm{p}}^{-2}$. Companions larger than the calculated limits should be detectable with an average $\mathrm{S} / \mathrm{N}$ of at least 5. For comparison we also show the size corresponding to the $1 \sigma$ photon noise limit. The radius limits in Fig. 7 are proportional to $(p(\alpha) \cdot I(\alpha))^{-1 / 2}$, therefore improving for planets with higher reflectivity and fractional polarisation.

\subsection{Contrast gain through longer integration}

One simple way of improving the achievable contrast limits is through longer integration $t_{\mathrm{exp}}$. Especially if photon noise dominates, the detection limits should be proportional to $t_{\exp }^{-1 / 2}$. At small separations from the star the noise is dominated by the noise residuals that were not eliminated perfectly in the PDI step. This can be seen for example in the bottom frame of Fig. A.2. Some of the aberrations - especially the ones to the right and left of the coronagraph caused by the deformable mirror (DM) are quasi-static throughout the observation (Cantalloube et al. 2019). This changes the statistics of the noise for smaller separations and can ultimately prevent the detection of a point-source signal with a reasonable $t_{\text {exp }}$.

In Fig. 8 we show how the polarimetric contrast evolves at different separations if we combine more and more polarimetric cycles. The points at $t_{\mathrm{exp}}=0.04 \mathrm{~min}$ show the contrast in a single zero-phase and $\pi$-phase combined $2 \times 1.2 \mathrm{~s}$ exposure as in the bottom frame of Fig. A.2. All other points show the polarimetric contrast in Stokes $Q$ from one single camera after combining the exposures of multiple polarimetric cycles. Here, PCA-ADI was not applied because the procedure requires a certain amount of field rotation to be effective, and therefore would make it difficult to directly compare the results for different total exposure times.

The data show that the noise for separations $\gtrsim 0.6^{\prime \prime}$ is proportional to $t_{\exp }^{-1 / 2}$, just as expected in the photon-noise-dominated regime, all the way from the shortest to the longest $t_{\exp }$, in perfect

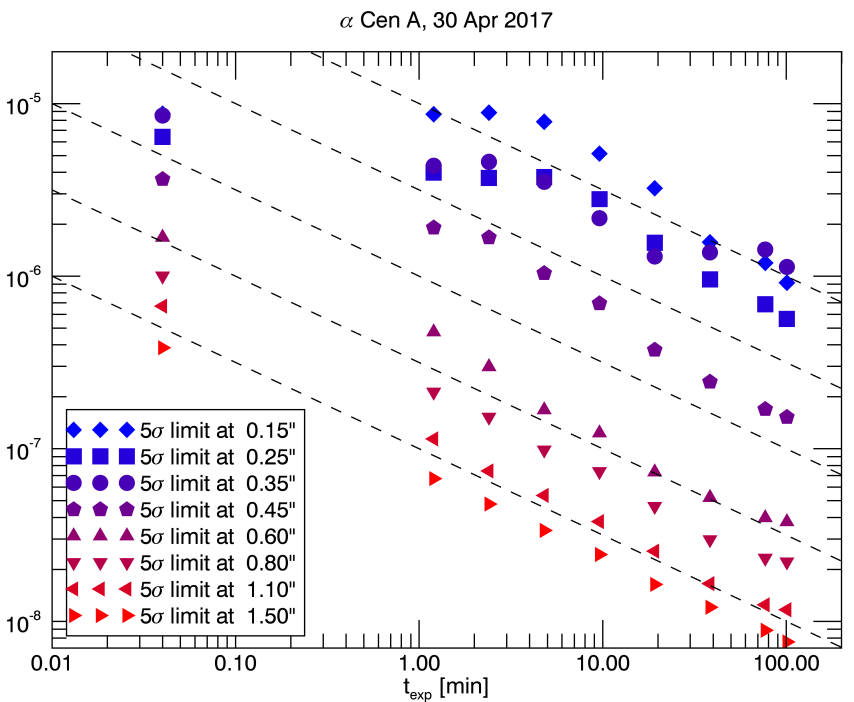

Fig. 8. Contrast limits at different $t_{\exp }$, calculated for a range of different separations from the star (indicated by different symbols). The dashed lines are proportional to $t_{\exp }^{-1 / 2}$, therefore emphasising the expected behaviour of the noise in the photon-noise-limited case.

agreement with what we see in the corresponding contrast curve (Fig. 6). This indicates for these separations that longer integrations would certainly improve the achievable contrast to a deeper level.

For small separations $\lesssim 0.6^{\prime \prime}$ and short integration times, the contrast first barely improves with increasing $t_{\text {exp }}$. Towards longer integration times however, it also changes to a $t_{\exp }^{-1 / 2} \mathrm{scal}-$ ing. The transition from a flat curve to a square-root scaling happens later for smaller separations. This can be explained because at small separations the noise is dominated by quasistatic aberrations, and therefore angular averaging by the field rotation increases the $\mathrm{S} / \mathrm{N}$ of a point source; nevertheless, the efficiency of this process depends on the separation and the speed of the field rotation. This explanation is supported by Fig. 8 where the transitions for the four separations $\rho=0.15^{\prime \prime}-$ $-0.45^{\prime \prime}$ happen when the field rotation leads to an azimuthal shift of about $1.5-2.5 \lambda / D$ at the corresponding separation. This corresponds to approximately the characteristic size of a speckle. As a reference, in the data used for this study, the speed of the field rotation during the relevant time period is $\sim 26 \mathrm{mas} / 10 \mathrm{~min}$ or $1.6 \lambda / D / 10 \mathrm{~min}$ at the observed wavelength.

\subsection{Detection limits}

The $\alpha$ Cen A/B system is a close binary with semimajor axis of 23.5 AU, which restricts the range of stable planetary orbits around the individual components. Wiegert \& Holman (1997) and Quarles \& Lissauer (2016) found that orbits around $\alpha$ Cen A are stable for semimajor axes up to $\sim 3$ AU. Stable orbits would preferably be coplanar to the binary orbital plane with inclination $i=79.2^{\circ}$ but deviations up to $\pm 45^{\circ}$ are not unlikely from a stability point of view. There are also reports of other massive planets around one component in close binary systems with a separation smaller than 25 AU (e.g. HD 196885 Correia et al. 2008, Gliese 86 Queloz et al. 2000; Lagrange et al. 2006, $\gamma$ Cep Hatzes et al. 2003; Neuhäuser et al. 2007, and HD 41004 A Zucker et al. 2004).

The radial velocity limits for $\alpha$ Cen A (e.g. Zhao et al. 2018) exclude the presence of massive planets with 
$M \sin (i)>53 M_{\text {Earth }}$ for the classically defined habitable zone from about 1 to $2 \mathrm{AU}$ with even more stringent limits for smaller separations. This evidence is not in favour of a planet around $\alpha$ Cen A with a mass larger than or comparable to Jupiter but planets up to almost $100 M_{\text {Earth }}$ cannot be excluded. Depending on the exact composition, formation history, and age, gas giants with masses like that could already be close to Jupiter sized (e.g. Swift et al. 2012).

With our radius limits in Fig. 7 we show that we might not be far from being able to detect a planet of this size around $\alpha$ Cen A. An important unknown factor in the radius limits are the reflective properties of the planet. For the limits in Fig. 7 we assumed a model with $Q\left(90^{\circ}\right)=0.055$ for the polarised reflectivity of the reflected light. This is optimistic for the reflection of stellar light by the atmosphere of a giant planet, but some models nevertheless predict even larger values. The combination of reflection and polarisation could also be larger due to other reasons. Calculations from Arnold \& Schneider (2004) have shown that a planet with a Saturn-like unresolved ring could have an exceptionally high brightness in reflected light.

It is not unreasonable to assume that $\alpha$ Cen A could harbour a still-undetected companion that could be observed with SPHERE/ZIMPOL in reflected visible light. Our best detection limits based on one single half-night show no evidence for a Jupiter-sized planet with exceptionally high fraction of polarised reflectivity. However, there is a temporal aspect to the detection limits because of the strong dependence of the reflected light intensity and polarisation fraction on the phase angle $\alpha$ (see Fig. 2); even a Jupiter-sized planet with exceptionally high reflection and polarisation would be faint for a large range of phase angles. Therefore, only a series of multiple observations could verify the absence of such a planet. Alternatively, one can carry out a detailed combined analysis of the detection limits and possible companion orbits for an estimate on the likelihood of observing a companion. We carried out an investigation like this for $\alpha$ Cen A and discuss the procedure and the results in Sect. 7.2.

As far as we know, there has not been a direct imaging search comparable to our study for planetary companions in reflected light around $\alpha$ Cen A. Kervella et al. (2006) performed an extensive direct imaging search for faint comoving companions around $\alpha \mathrm{Cen} \mathrm{A} / \mathrm{B}$ with NACO at the VLT in $J$-, $H$-, and $K$-band observations. However, their results are difficult to compare to ours because the IWA of their contrast limits is larger than the FOV of ZIMPOL. Schroeder et al. (2000) conducted a survey for low-mass stellar and substellar companions with the Hubble Space Telescope (HST) for some of the brightest stars closest to the Sun. The contrast limits of these authors for $\alpha$ Cen A in a range of separations $\left(0.5^{\prime \prime}-1.5^{\prime \prime}\right)$ are about $7.5-8.5 \mathrm{mag}$ at a wavelength of $\sim 1.02 \mu \mathrm{m}$. Our much deeper contrast limits in intensity are about 13.7-17.4 mag and in polarised intensity $18.3-20.4$ mag but with an effective IWA of only $\sim 0.13^{\prime \prime}$ for the $R$-band.

\section{Discussion}

We have shown the exceptional capability of SPHERE/ZIMPOL polarimetry for the search of reflected light from extrasolar planets on our prime target $\alpha$ Cen A in Sect. 6 and our additional targets in Appendix C. The combination of high resolution and polarimetric sensitivity of our observations is far superior to that of any other instrument. For $\alpha$ Cen A, B and Altair we derive polarimetric contrast limits better than 20 mag at separations $>1^{\prime \prime}$. Even at the effective coronagraphic IWA of $0.13^{\prime \prime}$ the polarimetric contrast limits can be around 16 mag. The same performance would also be possible for Sirius A during better observing conditions. A summary of the resulting $5 \sigma$ contrast limits for all targets can be found in Table 2. For the less bright objects $\epsilon$ Eri and $\tau$ Ceti we still see polarimetric contrast limits better than $18.9 \mathrm{mag}$ and $18.2 \mathrm{mag}$ at separations $>1^{\prime \prime}$, respectively, with 16 mag close to the effective IWA for $\tau$ Ceti. Photon-noise-limited polarimetric contrasts can be achieved already at separations as small as $0.6^{\prime \prime}$.

\subsection{Comparison to thermal infrared imaging}

Only a small number of other high-contrast direct imaging searches for planetary companions have been published for our targets (Schroeder et al. 2000; Kervella et al. 2006; Thalmann et al. 2011; Vigan et al. 2015; Mizuki et al. 2016; Boehle et al. 2019; Mawet et al. 2019). The observations were typically carried out with available NIR high-contrast imagers and the aim was usually to search for thermal light from brown dwarfs or very massive self-luminous planets. The detection of such objects around the nearest stars would have been possible, but is quite unexpected. For these NIR observations, the expected signal for the reflected light from a planet is far beyond our reach, but the obtained results represent the best limits achieved so far. The most sensitive limits were obtained with a combination of SDI and ADI for Sirius A with the SPHERE/IRDIFS mode (Vigan et al. 2015). Our observation of this object suffered from bad observing conditions; however, for PDI and ADI observations of similarly bright targets, our reported contrast limits show an improvement of 2-3 mag at all separations up to 1.7". However, much improved sensitivity is severely needed to detect a planet in reflected light. The only targets where a detection seems to be possible in a single night are $\alpha$ Cen $\mathrm{A}$ and $\mathrm{B}$. The lower brightness of the other targets decreases the sensitivity at a given angular separation and the larger distance to them increases the contrast of companions for the same angular separations.

The physical meaning of the contrast limits for the reflected light is different compared to the limits from IR surveys for the thermal emission from the planet. The contrast limits in the infrared probe the intrinsic luminosity and surface temperature and can be transformed into upper limits for the planet mass with models for planet formation and evolution (e.g. Baraffe et al. 2003; Spiegel \& Burrows 2012) if the age of the system is known and if the irradiation from the star can be neglected. Evolved planets are usually close to or at equilibrium temperature and emit for separations of $\sim 1 \mathrm{AU}$ or larger at longer wavelengths $(\sim 10 \mu \mathrm{m})$ where reaching high contrast is difficult with current ground-based observations. The intrinsic flux of planets drops off exponentially towards visible wavelengths. For example, assuming perfect black body spectra and a solarlike host star, even a self-luminous $800 \mathrm{~K}$ Jupiter-sized planet would only have a contrast of order $3 \times 10^{-11}$ in the visual $I$-band, while the contrast of the reflected light would be around $3 \times 10^{-8}$ for $d_{\mathrm{p}}<1 \mathrm{AU}$ and $I\left(90^{\circ}\right)=0.131$ (for a discussion on wavelength-dependent reflectivity see Sudarsky et al. 2003). Planets with $\mathrm{d}_{\mathrm{p}} \approx 1 \mathrm{AU}$ around $\alpha$ Cen A/B would have to be at temperatures above $\sim 1000 \mathrm{~K}$ to be brighter in thermal emission compared to reflected light at visible wavelengths. This is why we can only probe reflected stellar light in the visible wavelengths for all our targets and we do not expect any contribution from thermal emission.

The recently launched New Earths in the Alpha Cen Region (NEAR; Kasper et al. 2017; Käufl et al. 2018) survey using the VISIR instrument at the VLT aims to achieve high contrasts at $10 \mu \mathrm{m}$ for $\alpha \mathrm{Cen} \mathrm{A} / \mathrm{B}$ and to detect evolved planets in the 
S. Hunziker et al.: RefPlanets: search for reflected light from extrasolar planets with SPHERE/ZIMPOL

Table 2. Summary of $5 \sigma$ contrast limits for the intensity $C_{\text {flux }}$ and polarised intensity $C_{\mathrm{pol}}$ at some key separations for each target.

\begin{tabular}{|c|c|c|c|c|c|c|c|c|c|c|c|}
\hline \multirow[t]{2}{*}{ Object } & \multirow[t]{2}{*}{$m_{\mathrm{R}}$} & \multirow[t]{2}{*}{ Filters } & \multirow[t]{2}{*}{$t_{\exp }^{(a)}$} & \multicolumn{4}{|c|}{$\begin{array}{l}C_{\mathrm{pol}}(\mathrm{mag}) \\
\text { Inside } \mathrm{AO} \text { contr. rad. }{ }^{(b)}\end{array}$} & \multicolumn{4}{|c|}{$\begin{array}{r}C_{\text {flux }}(\mathrm{mag}) \\
\text { Inside AO contr. rad. }{ }^{(b)}\end{array}$} \\
\hline & & & & $\lesssim 0.35^{\prime \prime}$ & $\lesssim 0.45^{\prime \prime}$ & $0.5^{\prime \prime}$ & $1.5^{\prime \prime}$ & $\lesssim 0.35^{\prime \prime}$ & $\lesssim 0.45^{\prime \prime}$ & $0.5^{\prime \prime}$ & $1.5^{\prime \prime}$ \\
\hline Sirius A & -1.5 & N_I & $2 \mathrm{~h} 55.2 \mathrm{~min}$ & & 15.0 & 15.8 & 18.8 & & 11.0 & 11.8 & 15.7 \\
\hline Altair & 0.6 & R_PRIM & $2 \mathrm{~h} 31.2 \mathrm{~min}$ & 16.8 & & 17.9 & 20.4 & 12.7 & & 14.6 & 19.5 \\
\hline$\epsilon$ Eri & 3.0 & $\mathrm{VBB}$ & $3 \mathrm{~h} 12 \mathrm{~min}$ & 15.8 & & 16.2 & 19.6 & 10.4 & & 11.3 & 16.3 \\
\hline$\alpha$ Cen A & -0.5 & N_R & $3 \mathrm{~h} 21.6 \mathrm{~min}$ & 16.8 & & 18.3 & 20.4 & 12.8 & & 14.0 & 18.9 \\
\hline$\alpha$ Cen B & 1.0 & $\mathrm{VB} \bar{B}$ & $3 \mathrm{~h} 26.8 \mathrm{~min}$ & 17.1 & & 18.5 & 20.4 & 13.0 & & 14.1 & 18.5 \\
\hline$\tau$ Ceti & 2.9 & R_PRIM & $2 \mathrm{~h} 48 \mathrm{~min}$ & 15.8 & & 16.7 & 18.8 & 12.4 & & 13.7 & 18.2 \\
\hline
\end{tabular}

Notes. ${ }^{(a)}$ The combined total exposure time. ${ }^{(b)}$ Average value for the contrast limit at separations inside the AO control radius $(\lesssim 20 \lambda / D)$.

habitable zone of this binary system. The results of the NEAR campaign will be especially interesting for our survey since it will at least provide exceptionally deep detection limits in the IR that can be directly compared to our own limits for $\alpha$ Cen $\mathrm{A} / \mathrm{B}$ in reflected light at visible wavelengths.

\subsection{Interpreting the contrast limits}

In contrast to the thermal emission, the polarised intensity of reflected stellar light depends strongly on the planet radius $R_{\mathrm{P}}$, the planet-star separation $d_{\mathrm{p}}$, the reflective properties of the atmosphere and the phase of the planet (see Eq. (2)). Therefore, contrast limits yield - for a given physical separation, orbital phase, and reflective properties - an upper limit for the planet radius. This means that the upper limits for the size of a companion as presented for the $\alpha$ Cen A data come with a set of critical assumptions. The contrast limits are determined for the apparent separation between star and planet $\rho$. However, the reflected light brightness of the planet depends on the physical separation $d_{\mathrm{p}}$ and planets located at apparent separation $\rho$ can have any physical separation $d_{\mathrm{p}} \geq \rho$. This introduces a degeneracy into the calculation of physical parameters that cannot be lifted without further assumptions. For this reason, for the radius upper limits in Fig. 7 we assumed that the physical separation corresponds to the apparent separation, in addition to fixing the scattering model. This assumption can be justified for a blind search for planets with a Monte-Carlo (MC) simulation of apparent separations and contrasts for a random sample of planets. We simulated 5000000 Jupiter-sized planets on circular orbits around $\alpha$ Cen A with randomly distributed semi-major axes and inclinations and the Rayleigh scattering atmosphere model discussed in Sect. 2.2. We used a flat prior distribution for the orbital phase angles in the interval $[0,2 \pi]$ and for the semimajor axes in the interval $[0.01,3]$ AU. The inner boundary for the semi-major axis has a negligible effect on the final result as long as it is smaller than $0.18 \mathrm{AU}$ (the effective IWA of our data). Planets with larger semi-major axes would be unstable due to the close binary. For the inclination we assumed a Gaussian prior with a standard deviation of $45^{\circ}$, centred on the inclination of the binary orbit. Large mutual inclinations of binary and planetary orbits are unlikely due to stability reasons (Quarles \& Lissauer 2016). We chose $\alpha$ Cen A as our example because it has some of the best detection limits. Panel a in Fig. 9 shows the likelihood of one of the simulated planets having a certain apparent separation and contrast. The likelihood was calculated by dividing the number of MC samples in each contrast-separation bin by the total number of sampled planets. The likelihood drops to zero towards the upper right corner because planets at large separations have an upper limit on their reflected light intensity determined by their size and reflective properties. The dividing line with the strongly increased likelihood in the centre is $\propto \rho^{-2}$, representing planets at maximum elongation, corresponding to orbital phase angles close to $90^{\circ}$ and $270^{\circ}$. It is more likely for a planet to be located around this line, independent of the inclination of its orbit. For orbits close to edge-on, the apparent movement of the planet is slower at these phase angles. This naturally increases the likelihood of it being observed during this phase. For orbits closer to face-on the apparent separation of the planet will not change significantly during the orbit. This also increases the likelihood of the planet being observed during maximum elongation. Around $66 \%$ of all sampled planets end up inside the parameter space shown in Fig. 9 and 24\% end up in close proximity ( $\Delta m \approx 0.4 \mathrm{mag}$ ) to the line with maximum separation and contrast. This is a large fraction considering that we did not assume any prior knowledge about the orbital phase of the sample planets. We compare the likelihood to the completeness or the performance map (see Jensen-Clem et al. 2018) of our observation in panel b of Fig. 9, adopting the previously shown contrast curve for $\alpha$ Cen A (Fig. 6b) and assuming a Gaussian noise distribution. The full performance map in panel $b$ is drawn for a detection threshold $\tau=5 \sigma$ where we additionally show the $50 \%$ completeness contour for $\tau=3 \sigma$. The completeness can be understood as the fraction of true positives given $\tau=3 \sigma$ or $5 \sigma$. We multiply the performance map and likelihood in panels $\mathrm{c}$ and $\mathrm{d}$ in Fig. 9 to show the expected fraction of detectable planets for both detection thresholds and calculate the total integrated fraction of observed planets. Only about $1.5 \%$ of the samples would produce a signal with $S / N=5$ in our data but the number increases by almost a factor of ten to about $13.5 \%$ for signals with $S / N=3$. This happens because the shape of the contrast curve resembles the $\propto \rho^{-2}$ shape of the parameter space where the likelihood is strongly increased. If both curves are on a similar level in terms of contrast, just like in our case with $\alpha$ Cen A, a small contrast improvement can considerably increase the possibility of a detection. The same happens if we lower the detection threshold but this simultaneously increases the probability of a false detection (false-alarm probability) significantly. For Gaussian distributed noise and a $1024 \times 1024$ px $^{2}$ detector the expected number of random events exceeding $>5 \sigma$ is smaller than one, but the number increases to $\sim 1000$ for $>3 \sigma$. Therefore, the $5 \sigma$ threshold should definitely be respected in a blind search. However, a detection between 3 and $5 \sigma$ could be enough if there were multiple independent detections of this kind with ZIMPOL that could be combined into one single, more significant detection.

The MC simulation shows that the reflected light from a Jupiter-sized planet around $\alpha$ Cen A could be detected as a 

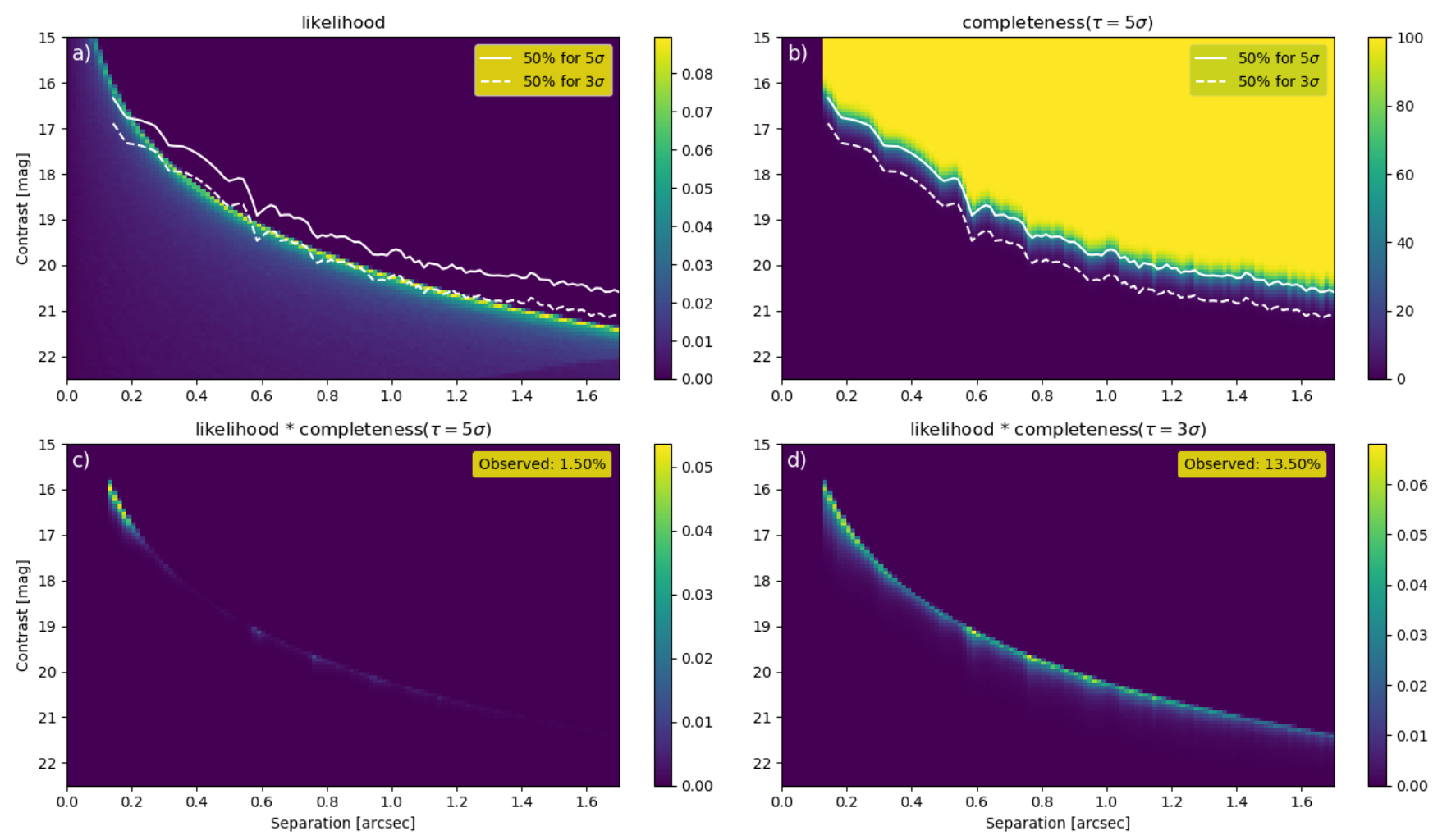

Fig. 9. Results of the Monte-Carlo sampling of 5000000 Jupiter-sized planets on circular orbits around $\alpha$ Cen A. Panel a: fraction of samples (in percent) that end up at a certain apparent separation and polarised intensity contrast at all times. Panel $b$ : full performance map for a certain detection threshold $N \tau$. This figure shows the probability of detecting an existing planet for all separations and contrasts with an $\mathrm{S} / \mathrm{N}$ of $N$. Panels $c$ and $d$ : combination of the likelihood and the performance map gives the fraction of planets at each separation and contrast that are detected as either $S / N=5$ or $S / N=3$ signals depending on the detection threshold.

$5 \sigma$ signal in a single half-night, when it is located relatively close $\left(0.13^{\prime \prime}-0.3^{\prime \prime}\right)$ to the star. It should also be possible to detect Jupiter-sized planets at any other separation as $3 \sigma$ signals and multiple $3 \sigma$ detections could be combined to a $5 \sigma$ result. Alternatively, a less significant detection could be considered sufficient if the position of the planet is known from another high-contrast detection or from the astrometric reflex motion of the star.

\subsection{Improving the contrast limits}

There are multiple ways to improve the detection limits with ZIMPOL with future observations. Different strategies are required for blind searches when compared to follow up observations of already known planets. For blind searches, the most effective way is to just increase the total integration time. We show in Sect. 6.4 that the contrast improves with the square root of the integration time. The observations should be done in P1 polarimetry mode to enable ADI to improve the contrast at smaller separations. For longer total integration times it will be necessary to combine the data from multiple observing nights. This is not straight forward for our targets because the apparent orbital motion is large. The most extreme case is $\alpha$ Cen A for which a planet on a face-on circular orbit would move 40 mas or $\sim 2 \lambda / \mathrm{D}$ per day at the IWA of $0.13^{\prime \prime}$ and 10 mas or $\sim 0.5 \lambda / \mathrm{D}$ at $1.7^{\prime \prime}$. For the combination of data from different, even consecutive nights it will be necessary to consider the Keplerian motion of planets. This is possible with data-analysis tools like K-Stacker (Nowak et al. 2018). K-Stacker was developed especially for finding weak planet signals in a time series of images when they move on Keplerian orbits. For a time series spanning a number of weeks it would also be necessary to additionally consider the change of the reflected polarised intensity as a function of orbital phase (Fig. 2). The orbital motion of planets around nearby stars could also be used as an advantage to further improve the contrast limits. Males et al. (2015) developed the concept of orbital differential imaging (ODI) that exploits the orbital motion of a planet in multi-epoch data to remove the stellar PSF while minimizing the subtraction of the planet signal.

Follow-up observations of a known planet would confer major advantages over a blind search because the prior knowledge of orbital phase or orbit location from RV or astrometric measurements can be exploited for optimizing the observing strategy and simplifying the analysis of the data. Currently, the best planets for a successful follow up with ZIMPOL are the giant planets $\epsilon$ Eri b and GJ $876 \mathrm{~b}$ and the terrestrial planet Proxima Centauri b. The planet around $\epsilon$ Eri can be observed at the favourable photon-noise-limited apparent separation of $\sim 0.8^{\prime \prime}$ with ZIMPOL. However, it is expected to be rather faint in reflected light because of its large semi-major axis of $\sim 3 \mathrm{AU}$. The polarimetric contrasts of both Proxima Centauri b and GJ 876 b are expected to be less demanding but the expected maximum separation of only $\sim 0.04^{\prime \prime}$, corresponding to $\sim 2 \lambda / \mathrm{D}$ in the visible, is very challenging. This requires a specialised instrumental setup for SPHERE/ZIMPOL, such as for example an optimised pupil mask developed to suppress the first Airy ring at $2 \lambda / \mathrm{D}$ as proposed by Patapis et al. (2018).

For companions with known separation, the selection of the ZIMPOL instrument mode can also be optimised. The P1 polarimetry mode should be used for companions close to or 
inside the AO control ring $<0.7^{\prime \prime}$ because it allows the use of ADI for additional speckle noise suppression. Angular differential imaging also helps to reduce static noise induced by the instrument itself. However, for larger separations ADI is not necessary and the field stabilised P2 polarimetry mode could be used. This would allow the use of longer DIT without diluting the planet signal due to the field rotation during exposures.

For planets with well-known orbital parameters such as semimajor axis, inclination, and orbital phase, it would be possible to plan observations to be executed at the right time when the reflected intensity and apparent separation are optimal. Finally, if also the position angle of the orbit were known, it would be possible to align the polarimetric $Q$-direction of ZIMPOL with the expected orientation of the polarised signal from the planet. This would allow us to only observe in a rotated $Q$ polarisation coordinate system without spending half of the time observing $U$, which is expected to be zero. The observation time would be cut in half for the same detection sensitivity or the contrast limit would be improved by a factor of $\sqrt{2}$ in the same amount of telescope time.

There are certainly other ways to improve the detection limits which have not yet been sufficiently investigated. The use of narrowband versus broadband filters could be beneficial because instrumental effects like beam shift and instrumental polarisation are wavelength dependent and the post-processing cannot fully account for this. Therefore, the applied corrections are not optimal for observations taken with broadband filters and would provide better results for narrowband filters. Another way to improve the detection limits is frame selection. The gain from both of the mentioned techniques is difficult to quantify because we did not find any point sources in our data. Adding more data, even data of poor quality, generally improved the calculated detection limits because it decreased the noise level of the data. However, data of poor quality also lower the signal of a point source but this effect can only be studied properly if a real signal is present in the data because for deep coronagraphic observations we do not know the exact PSF shape for each image.

\section{Conclusions}

We observed $\alpha$ Cen A and B, Sirius A, $\epsilon$ Eri, and $\tau$ Cet using SPHERE/ZIMPOL in polarimetry mode. The target list for the search of reflected light from extrasolar planets with direct imaging is short and the targets were selected for achieving deep detection limits within a few hours of observations. We were not able to detect a polarised intensity signal above the detection threshold from any of our targets. However, our data provide some of the deepest contrast limits for direct imaging to date. The achieved limits for our brightest targets show that the detection of polarised reflected light from a $1 R_{\mathrm{J}}$-sized object would be possible in a single night under good observing conditions (Seeing $\lesssim 0.8^{\prime \prime}, \tau_{0} \gtrsim 4 \mathrm{~ms}$ ) for our nearest neighbours, $\alpha$ Cen A/B, with a realistic model for a reflecting atmosphere. Unfortunately, our null result is not constraining for the occurrence rate of giant planets because of the strong time dependence of the reflected light intensity, and given the low frequency of gas giants with 110 Jupiter masses between 0.3 and $3 \mathrm{AU}$ this rate is expected to be only about 4\% (Cumming et al. 2008; Fernandes et al. 2019), slightly higher for A-stars (Johnson et al. 2010), but lower for intermediate-separation binaries (Kraus et al. 2016).

Our results show the capability of ZIMPOL to remove the unpolarised stellar PSF and to deliver the deepest contrast limits for direct imaging at visible wavelengths from 600 to $900 \mathrm{~nm}$. The performance is close to the photon noise limit and this allows the contrast limits to be scaled for different total integration times and for targets with different brightnesses. This will be useful in the future for planning further observations, in particular for larger programs with deeper observations of the surroundings of the nearest stars by combining the results of many nights. Due to the strong phase dependence, the search for reflected light is especially suitable for potential follow-up observation of targets with known orbital phases already determined with different methods (e.g. RV and astrometry). Another use of the highly sensitive polarimetry with ZIMPOL could be the determination of the linear polarisation of the thermal light from low-mass companions. This measurement has been tried before for a few different targets at infrared wavelengths (e.g. Jensen-Clem et al. 2016; van Holstein et al. 2017). The main difficulty with brown dwarf companions is that the linear polarisation degree for the thermal light is expected to be $<1 \%$ (Stolker et al. 2017). Another problem for ZIMPOL polarimetry is the low luminosity of $\mathrm{L}$ and $\mathrm{T}$ dwarfs in the visible wavelengths.

Another important aspect of this work is our investigation of the limitations of SPHERE/ZIMPOL at the VLT. We analysed and corrected the residual instrument polarisation and most importantly the polarimetric beam-shift effect. The beam-shift effect is well known in optics but ZIMPOL is the first astronomical instrument where this effect is apparent in the data because of its high spatial resolution and polarimetric sensitivity. Despite all the calibrations and corrections applied in this work, there remain substantial speckle residuals in the differential polarimetry which currently limit the contrast performance at small separations $\left(<0.6^{\prime \prime}\right)$. However, the achieved polarimetric contrast $C_{\text {pol }}$ is in most cases more than ten times deeper for separations $<1^{\prime \prime}$ and up to approximately 50 times deeper at the smallest separations compared to the imaging contrast $C_{\text {flux }}$ achieved with classical PCA-ADI processing. Over a larger range of separations, deep polarimetric contrast limits and even photon-noise-limited performance is achieved without additional PCA-ADI. Therefore, polarimetry is a method of utmost potential for pushing the detection limits of reflecting planets with future high-contrast instruments (Kasper et al. 2010). In particular, speckle suppression can be further improved by better avoiding colour-dependent disturbing effects (see Appendix A) or by taking advantage of the much improved light-gathering power of the upcoming generation of 30-40m ELTs. With larger telescopes it will be possible to achieve the same contrast limits for fainter stars at higher resolution, significantly increasing the sample size of nearby targets and possibly allowing the planets in the habitable zone of nearby $M$ dwarfs to be imaged, such as Proxima Centauri for example. The experience gained with the SPHERE/ZIMPOL RefPlanets survey described in this work should therefore be helpful for design studies and the development of such instruments.

Acknowledgements. S.H. and H.M.S. acknowledge the financial support by the Swiss National Science Foundation through grant 200020_162630/1. SPHERE is an instrument designed and built by a consortium consisting of IPAG (Grenoble, France), MPIA (Heidelberg, Germany), LAM (Marseille, France), LESIA (Paris, France), Laboratoire Lagrange (Nice, France), INAF-Osservatorio di Padova (Italy), Observatoire de Genève (Switzerland), ETH Zurich (Switzerland), NOVA (Netherlands), ONERA (France) and ASTRON (Netherlands) in collaboration with ESO. SPHERE was funded by ESO, with additional contributions from CNRS (France), MPIA (Germany), INAF (Italy), FINES (Switzerland) and NOVA (Netherlands). SPHERE also received funding from the European Commission Sixth and Seventh Framework Programmes as part of the Optical Infrared Coordination Network for Astronomy (OPTICON) under grant number RII3-Ct-2004-001566 for FP6 (2004-2008), grant number 226604 for FP7 (2009-2012) and grant number 312430 for FP7 (2013-2016). We also acknowledge financial support from the Programme National de Planétologie (PNP) and the Programme National de Physique Stellaire (PNPS) of CNRS-INSU in 
France. This work has also been supported by a grant from the French Labex OSUG@2020 (Investissements d'avenir - ANR10 LABX56). The project is supported by CNRS, by the Agence Nationale de la Recherche (ANR-14-CE330018). It has also been carried out within the frame of the National Centre for Competence in Research PlanetS supported by the Swiss National Science Foundation (SNSF). M.R.M. and S.P.Q. are pleased to acknowledge this financial support of the SNSF. Finally, this work has made use of the SPHERE Data Centre, jointly operated by OSUG/IPAG (Grenoble), PYTHEAS/LAM/CESAM (Marseille), OCA/Lagrange (Nice), Observatoire de Paris/LESIA (Paris), and Observatoire de Lyon, also supported by a grant from Labex OSUG@2020 (Investissements d'avenir - ANR10 LABX56). We thank P. Delorme and E Lagadec (SPHERE Data Centre) for their efficient help during the data reduction process. This research has made use of the SIMBAD database, operated at CDS Strasbourg, France. This work has been partially supported by the project PRININAF 2016 The Cradle of Life- GENESIS-SKA (General Conditions in Early Planetary Systems for the rise of life with SKA). We also acknowledge support from INAF/Frontiera (Fostering high ResolutiON Technology and Innovation for Exoplanets and Research in Astrophysics) through the "Progetti Premiali" funding scheme of the Italian Ministry of Education, University, and Research. T.H acknowledges support from the European Research Council under the Horizon 2020 Framework Program via the ERC Advanced Grant Origins 832428 .

\section{References}

Amara, A., \& Quanz, S. P. 2012, MNRAS, 427, 948

Arnold, L., \& Schneider, J. 2004, A\&A, 420, 1153

Backman, D., Marengo, M., Stapelfeldt, K., et al. 2009, ApJ, 690, 1522

Bailey, J., Lucas, P. W., \& Hough, J. H. 2010, MNRAS, 405, 2570

Bailey, J., Cotton, D. V., \& Kedziora-Chudczer, L. 2017, MNRAS, 465, 1601

Bailey, J., Kedziora-Chudczer, L., \& Bott, K. 2018, MNRAS, 480, 1613

Baraffe, I., Chabrier, G., Barman, T. S., Allard, F., \& Hauschildt, P. H. 2003, A\&A, 402, 701

Bazzon, A., Gisler, D., Roelfsema, R., et al. 2012, Proc. SPIE, 8446, 844693

Bazzon, A., Schmid, H. M., \& Gisler, D. 2013, A\&A, 556, A117

Bazzon, A., Schmid, H. M., \& Buenzli, E. 2014, A\&A, 572, A6

Beuzit, J.-L., Feldt, M., Dohlen, K., et al. 2008, SPIE Conf. Ser., 7014, 18

Beuzit, J. L., Vigan, A., Mouillet, D., et al. 2019, A\&A, 631, A155

Boehle, A., Quanz, S. P., Lovis, C., et al. 2019, A\&A, 630, A50

Bond, H. E., Schaefer, G. H., Gilliland, R. L., et al. 2017, ApJ, 840, 70

Booth, M., Dent, W. R. F., Jordán, A., et al. 2017, MNRAS, 469, 3200

Bottom, M., Ruane, G., \& Mawet, D. 2017, Res. Notes AAS, 1, 30

Bowler, B. P. 2016, PASP, 128, 102001

Buenzli, E., \& Schmid, H. M. 2009, A\&A, 504, 259

Cantalloube, F., Dohlen, K., Milli, J., Brandner, W., \& Vigan, A. 2019, The Messenger, 176, 25

Chauvin, G., Desidera, S., Lagrange, A. M., et al. 2017, A\&A, 605, L9

Claudi, R. U., Turatto, M., Gratton, R. G., et al. 2008, SPIE Conf. Ser., 7014 70143E

Correia, A. C. M., Udry, S., Mayor, M., et al. 2008, A\&A, 479, 271

Cotton, D. V., Bailey, J., Kedziora-Chudczer, L., et al. 2016, MNRAS, 455, 1607

Cotton, D. V., Bailey, J., Howarth, I. D., et al. 2017a, Nat. Astron., 1, 690

Cotton, D. V., Marshall, J. P., Bailey, J., et al. 2017b, MNRAS, 467, 873

Cumming, A., Butler, R. P., Marcy, G. W., et al. 2008, PASP, 120, 531

Dohlen, K., Langlois, M., Saisse, M., et al. 2008, SPIE Conf. Ser., 7014, 70143L

Dollfus, A. 1985, Adv. Space Res., 5, 47

Feng, F., Tuomi, M., Jones, H. R. A., et al. 2017, AJ, 154, 135

Fernandes, R. B., Mulders, G. D., Pascucci, I., Mordasini, C., \& Emsenhuber, A. 2019, ApJ, 874, 81

Fusco, T., Petit, C., Rousset, G., et al. 2006, SPIE Conf. Ser., 6272, 62720

Gisler, D., Schmid, H. M., Thalmann, C., et al. 2004, SPIE Conf. Ser., 5492, 463

Greaves, J. S., Wyatt, M. C., Holland, W. S., \& Dent, W. R. F. 2004, MNRAS, 351, L54

Greaves, J. S., Sibthorpe, B., Acke, B., et al. 2014, ApJ, 791, L11

Hansen, J. E., \& Hovenier, J. W. 1974, J. Atm. Sci., 31, 1137

Hatzes, A. P., Cochran, W. D., McArthur, B., et al. 2000, ApJ, 544, L145

Hatzes, A. P., Cochran, W. D., Endl, M., et al. 2003, ApJ, 599, 1383

Holman, M. J., \& Wiegert, P. A. 1999, AJ, 117, 621

Jensen-Clem, R., Millar-Blanchaer, M., Mawet, D., et al. 2016, ApJ, 820, 111

Jensen-Clem, R., Mawet, D., Gomez Gonzalez, C. A., et al. 2018, AJ, 155, 19

Johnson, J. A., Aller, K. M., Howard, A. W., \& Crepp, J. R. 2010, PASP, 122, 905

Jovanovic, N., Martinache, F., Guyon, O., et al. 2015, PASP, 127, 890

Kasper, M., Beuzit, J.-L., Verinaud, C., et al. 2010, Proc. SPIE, 7735, 77352E

Kasper, M., Arsenault, R., Käufl, H. U., et al. 2017, The Messenger, 169, 16

Käufl, H.-U., Kasper, M., Arsenault, R., et al. 2018, Proc. SPIE Conf. Ser., 10702, 107020D
Keller, C. U., Schmid, H. M., Venema, L. B., et al. 2010, Proc. SPIE, 7735, $77356 \mathrm{G}$

Kemp, J. C., Henson, G. D., Steiner, C. T., \& Powell, E. R. 1987, Nature, 326, 270

Keppler, M., Benisty, M., Müller, A., et al. 2018, A\&A, 617, A44

Kervella, P., Thévenin, F., Coudé du Foresto, V., \& Mignard, F. 2006, A\&A, 459, 669

Kervella, P., Mignard, F., Mérand, A., \& Thévenin, F. 2016, A\&A, 594, A107

Kervella, P., Arenou, F., Mignard, F., \& Thévenin, F. 2019, A\&A, 623, A72

Kraus, A. L., Ireland, M. J., Huber, D., Mann, A. W., \& Dupuy, T. J. 2016, AJ, 152,8

Lagrange, A. M., Beust, H., Udry, S., Chauvin, G., \& Mayor, M. 2006, A\&A, 459,955

Lagrange, A.-M., Desort, M., Galland, F., Udry, S., \& Mayor, M. 2009, A\&A, 495, 335

Lawler, S. M., Di Francesco, J., Kennedy, G. M., et al. 2014, MNRAS, 444, 2665

Macintosh, B., Graham, J. R., Ingraham, P., et al. 2014, Proc. Natl. Acad. Sci., 111,12661

Macintosh, B., Graham, J. R., Barman, T., et al. 2015, Science, 350, 64

Males, J. R., Belikov, R., \& Bendek, E. 2015, SPIE Conf. Ser., 9605, 960518

Marois, C., Macintosh, B., Barman, T., et al. 2008, Science, 322, 1348

Mawet, D., Milli, J., Wahhaj, Z., et al. 2014, ApJ, 792, 97

Mawet, D., Hirsch, L., Lee, E. J., et al. 2019, AJ, 157, 33

McLean, W., Stam, D. M., Bagnulo, S., et al. 2017, A\&A, 601, A142

Milli, J., Mouillet, D., Mawet, D., et al. 2013, A\&A, 556, A64

Mizuki, T., Yamada, T., Carson, J. C., et al. 2016, A\&A, 595, A79

Monnier, J. D., Zhao, M., Pedretti, E., et al. 2007, Science, 317, 342

Neuhäuser, R., Mugrauer, M., Fukagawa, M., Torres, G., \& Schmidt, T. 2007, A\&A, 462, 777

Nowak, M., Le Coroller, H., Arnold, L., et al. 2018, A\&A, 615, A144

Patapis, P., Kühn, J., \& Schmid, H. M. 2018, SPIE Conf. Ser., 10706, 107065J

Pavlov, A., Möller-Nilsson, O., Feldt, M., et al. 2008, Proc. SPIE, 7019, 701939

Quarles, B., \& Lissauer, J. J. 2016, AJ, 151, 111

Queloz, D., Mayor, M., Weber, L., et al. 2000, A\&A, 354, 99

Schmid, H. M., Beuzit, J.-L., Feldt, M., et al. 2006a, in IAU Colloq. 200: Direct

Imaging of Exoplanets: Science \& Techniques, ed. C. Aime \& F. Vakili, 165

Schmid, H. M., Joos, F., \& Tschan, D. 2006b, A\&A, 452, 657

Schmid, H. M., Joos, F., Buenzli, E., \& Gisler, D. 2011, Icarus, 212, 701

Schmid, H.-M., Downing, M., Roelfsema, R., et al. 2012, SPIE, 8446, 8

Schmidt, T. O. B., Neuhäuser, R., Briceño, C., et al. 2016, A\&A, 593, A75

Schmid, H. M., Bazzon, A., Roelfsema, R., et al. 2018, A\&A, 619, A9

Schroeder, D. J., Golimowski, D. A., Brukardt, R. A., et al. 2000, AJ, 119, 906

Schworer, G., \& Tuthill, P. G. 2015, A\&A, 578, A59

Seager, S., Whitney, B. A., \& Sasselov, D. D. 2000, ApJ, 540, 504

Smith, P. H., \& Tomasko, M. G. 1984, Icarus, 58, 35

Spiegel, D. S., \& Burrows, A. 2012, ApJ, 745, 174

Stam, D. M. 2008, A\&A, 482, 989

Stam, D. M. Hovenier, J. W., \& Waters, L. B. F. M. 2004, A\&A, 428, 663

Stolker, T., Min, M., Stam, D. M., et al. 2017, A\&A, 607, A42

Stolker, T., Bonse, M. J., Quanz, S. P., et al. 2019, A\&A, 621, A59

Sudarsky, D., Burrows, A., \& Hubeny, I. 2003, ApJ, 588, 1121

Swift, D. C., Eggert, J. H., Hicks, D. G., et al. 2012, ApJ, 744, 59

Thalmann, C., Schmid, H. M., Boccaletti, A., et al. 2008, SPIE Conf. Ser., 7014, 3

Thalmann, C., Janson, M., Buenzli, E., et al. 2011, ApJ, 743, L6

Tomasko, M. G., \& Smith, P. H. 1982, Icarus, 51, 65

Tuomi, M., Jones, H. R. A., Jenkins, J. S., et al. 2013, A\&A, 551, A79

van Dam, M. A., Le Mignant, D., \& Macintosh, B. A. 2004, Appl. Opt., 43, 5458

van Holstein, R. G., Snik, F., Girard, J. H., et al. 2017, SPIE Conf. Ser., 10400, 1040015

Vigan, A., Gry, C., Salter, G., et al. 2015, MNRAS, 454, 129

Wiegert, P. A., \& Holman, M. J. 1997, AJ, 113, 1445

Zhao, L., Fischer, D. A., Brewer, J., Giguere, M., \& Rojas-Ayala, B. 2018, AJ, 155,24

Zucker, S., Mazeh, T., Santos, N. C., Udry, S., \& Mayor, M. 2004, A\&A, 426, 695

1 ETH Zurich, Institute for Particle Physics and Astrophysics, Wolfgang-Pauli-Strasse 27, CH-8093 Zurich, Switzerland e-mail: silvan.hunziker@phys .ethz.ch

2 NOVA Optical Infrared Instrumentation Group at ASTRON, Oude Hoogeveensedijk 4, 7991 PD Dwingeloo, The Netherlands 
${ }^{3}$ Université Grenoble Alpes, IPAG, 38000 Grenoble, France

${ }^{4}$ CNRS, IPAG, 38000 Grenoble, France

5 European Southern Observatory, Alonso de Cordova 3107, Casilla 19001 Vitacura, Santiago 19, Chile

${ }^{6}$ Institute for Computational Science, University of Zürich, Winterthurerstrasse 190, 8057 Zürich, Switzerland

7 Istituto Ricerche Solari Locarno, Via Patocchi 57, 6605 Locarno Monti, Switzerland

8 Kiepenheuer-Institut für Sonnenphysik, Schneckstr. 6, 79104 Freiburg, Germany

9 Anton Pannekoek Institute for Astronomy, University of Amsterdam, PO Box 94249, 1090 GE Amsterdam, The Netherlands

${ }^{10}$ LESIA, CNRS, Observatoire de Paris, Université Paris Diderot, UPMC, 5 place J. Janssen, 92190 Meudon, France

${ }^{11}$ Leiden Observatory, Leiden University, PO Box 9513, 2300 RA Leiden, The Netherlands

12 Laboratoire Lagrange, UMR7293, Université de Nice SophiaAntipolis, CNRS, Observatoire de la Côte d'Azur, Boulevard de l'Observatoire, 06304 Nice, Cedex 4, France

${ }_{13}$ Max-Planck-Institut für Astronomie, Königstuhl 17, 69117 Heidelberg, Germany

${ }^{14}$ INAF - Osservatorio Astronomico di Padova, Vicolo dell'Osservatorio 5, 35122 Padova, Italy
15 Aix-Marseille Université, CNRS, CNES, LAM (Laboratoire d'Astrophysique de Marseille) UMR 7326, 13388, Marseille, France

16 Unidad Mixta International Franco-Chilena de Astronomia, CNRS/INSU UMI 3386 and Departemento de Astronomia, Universidad de Chile, Casilla 36-D, Santiago, Chile

${ }_{17}$ Nucleo de Astronomia, Facultad de Ingenieria y Ciencias, Universidad Diego Portales, Av. Ejercito 441, Santiago, Chile

18 Escuela de Ingenieria Industrial, Facultad de Ingenieria y Ciencias, Universidad Diego Portales, Av. Ejercito 441, Santiago, Chile

19 European Southern Observatory, Karl Schwarzschild St, 2, 85748 Garching, Germany

${ }^{20}$ ONERA, The French Aerospace Lab BP72, 29 avenue de la Division Leclerc, 92322 Châtillon Cedex, France

${ }^{21}$ Centre de Recherche Astrophysique de Lyon, CNRS/ENSL Université Lyon 1, 9 av. Ch. André, 69561 Saint-Genis-Laval, France

22 Geneva Observatory, University of Geneva, Chemin des Mailettes 51, 1290 Versoix, Switzerland

${ }^{23}$ STAR Institute, Université de Liège, Allée du Six Août 19c, 4000 Liège, Belgium

${ }^{24}$ Space Telescope Science Institute, 3700 St Martin Drive, Baltimore, MD, USA

${ }^{25}$ Department of Astronomy, University of Michigan, Ann Arbor, MI 48109, USA 


\section{Appendix A: Advanced data-reduction steps}

\section{A.1. Frame-transfer smearing correction}

ZIMPOL uses frame-transfer CCDs which shift each frame at the end of an illumination from the image area to a covered read-out area of the detector. There, the previously illuminated frame is read out during integration of the subsequent frame. The detector is also illuminated during the fast frame transfer, which lasts $56 \mathrm{~ms}$ for fast polarimetry, and this causes a frame-transfer smearing of the frame in the column direction. The smearing amounts to a maximum of $5 \%$ for the shortest integration time of $1.1 \mathrm{~s}$ and less for longer integrations (see Schmid et al. 2018, 2012). The smearing corrects itself in the polarisation signal $Q^{Z}$ but is apparent in the intensity signal $I^{Z}$ for short exposures. We correct this frame-transfer smearing in each intensity image with the subtraction of a correctly scaled mean row profile from every row in the bias-subtracted image.

\section{A.2. Telescope polarisation correction}

The SPHERE/ZIMPOL instrument uses a half wave plate (HWP) polarisation switch to select opposite polarisation modes $Q^{+}$and $Q^{-}$, or $U^{+}$and $U^{-}$to compensate the instrumental polarisation (Bazzon et al. 2012). However, there is a remaining residual telescope polarisation $p_{\text {tel }}$ from the optical components located in front of the HWP switch. The magnitude of the residual telescope polarisation $p_{\text {tel }}^{2}=p_{\text {tel }, Q}^{2}\left(\theta_{\mathrm{par}}\right)+p_{\mathrm{tel}, U}^{2}\left(\theta_{\mathrm{par}}\right)$ is wavelength dependent but otherwise essentially constant. The orientation of $p_{\text {tel }}$ rotates in the Stokes $Q-U$ plane as a function of the parallactic angle of the telescope $\theta_{\text {par }}$. The measured Stokes parameters $Q_{\mathrm{obs}}$ and $U_{\mathrm{obs}}$ are therefore a combination of the astrophysical polarisation of the target $Q, U$ and the induced telescope polarisation:

$Q_{\text {obs }}(x, y)=Q(x, y)+p_{\text {tel }, Q}\left(\theta_{\mathrm{par}}\right) \cdot I(x, y)$

$U_{\mathrm{obs}}(x, y)=U(x, y)+p_{\mathrm{tel}, U}\left(\theta_{\mathrm{par}}\right) \cdot I(x, y)$.

To first order, the telescope polarisation does not depend on the image coordinates $(x, y)$. Therefore, we can estimate the fractional telescope polarisation for unpolarised sky sources $(Q(x, y) \approx 0$ and $U(x, y) \approx 0)$ by measuring the mean ${ }^{2}$ fractional polarisation $Q_{\mathrm{obs}} / I$ and $U_{\mathrm{obs}} / I$ :

$p_{\text {tel }, Q} \approx\left\langle Q_{\text {obs }}(x, y)\right\rangle /\langle I(x, y)\rangle$

$p_{\mathrm{tel}, U} \approx\left\langle U_{\mathrm{obs}}(x, y)\right\rangle /\langle I(x, y)\rangle$.

Schmid et al. (2018) used measurements of unpolarised standard stars for the determination of the amplitude $p_{\text {tel }}$ in several different filters for SPHERE/ZIMPOL, which can be described by

$p_{\text {tel }, Q}\left(\theta_{\text {par }}\right)=p_{\text {tel }} \cdot \cos \left(2\left(\theta_{\text {par }}+\delta_{\text {tel }}\right)\right)$,

$p_{\text {tel }, U}\left(\theta_{\text {par }}\right)=p_{\text {tel }} \cdot \sin \left(2\left(\theta_{\text {par }}+\delta_{\text {tel }}\right)\right)$,

where $\delta_{\text {tel }}$ is a wavelength-dependent offset angle for the telescope polarisation.

2 Average over pixels in a ring around the star with inner radius $r$ larger than the coronagraph and outer radius $R$ :

$\langle f(x, y)\rangle=\frac{\sum_{r^{2}<x^{2}+y^{2}<R^{2}} f(x, y)}{\sum_{r^{2}<x^{2}+y^{2}<R^{2}} 1}$.
Our targets are not all zero-polarisation standard stars, but previous measurements have shown little intrinsic polarisation for any of them. High-precision polarimetry has resulted in measurements of $15 \times 10^{-6}$ for $\alpha$ Cen (Bailey et al. 2017) and $6 \times 10^{-6}$ for Sirius (Cotton et al. 2016). Measurements for the active star $\epsilon$ Eri have shown a polarisation of $30 \times 10^{-6}$ (Cotton et al. 2017b). For the debris disk host star $\tau$ Ceti, the measurements are consistent with zero polarisation (Cotton et al. 2017b). Altair could be an exception and it may show larger intrinsic linear polarisation because it is a rapidly rotating and, therefore, ellipsoidal A-star (Monnier et al. 2007). The expected linear polarisation of rapidly rotating early type stars was observed for the first time with the high-precision polarimetry of Regulus by Cotton et al. (2017a). However, the linear polarisation of Altair is expected to be lower than Regulus, and indeed existing polarimetry of Altair yields a low polarisation of $<10^{-5}$ (Bailey et al. 2010). No interstellar polarisation component is expected for our stars because of the distance of only a few parsecs, as is confirmed by high-precision polarimetry (Bailey et al. 2010; Cotton et al. 2016). Therefore, with our current polarimetric accuracy, we do not expect to measure any intrinsic polarisation that is inconsistent with zero for our targets.

Because our targets were observed over a large range of parallactic angles, they show the steady rotation of $p_{\text {tel }}$ as illustrated in Fig. A.1 for the narrow $R$-band observations of $\alpha$ Cen A taken on April 30, 2017. Each point in the plot shows the average telescope polarisation measured in an annulus - extending from $0.13^{\prime \prime}$ to $0.72^{\prime \prime}$ - in the stellar PSF halo centred on the star. The data can be well fitted with the model in Eq. (A.3) with $p_{\text {tel }}=0.12 \%$ and $\delta_{\text {tel }}=88.4^{\circ}$. The residuals indicate that the instrumental second-order effects and the intrinsic polarisation of $\alpha$ Cen A are of order $\lesssim 0.02 \%$ and also indicate a possible systematic shift of the polarisation towards positive $U$ values in Fig. A.1. However, fitting a model with a systematic shift as additional free parameter does not significantly improve the residuals. From this we conclude that the majority of the residuals are caused by instrumental effects.

In our $Q$ and $U$ differential polarisation image, the instrument polarisation produces a faint copy of the intensity images at the level of $0.1 \%$ (see Fig. A.2, top). We correct this by measuring $p_{\text {tel, } Q}$ and $p_{\text {tel }, U}$ (Eq. (A.2)) and subtracting the scaled intensity frames $p_{\mathrm{tel}, Q} \cdot I, p_{\mathrm{tel}, U} \cdot I$ from the corresponding $Q$ and $U$ frames. Figure A. 2 shows the polarised intensity before (top panel) and after (bottom panel) the subtraction.

The instrument polarisation is not a dominating source of noise for the search of a localized point source. Our analysis of the noise after different reduction and calibration steps (see Fig. 3) shows only small improvements for most separations. This is understandable if we separate the noise in a $Q_{\text {obs }}$ frame with instrument polarisation, as defined in Eq. (A.1), into its two main contributions $\delta Q$ and $p_{\text {tel }} \cdot \delta I$. The noise of the polarised intensity $\delta Q$ originates mainly from residuals of the PDI speckle cancellation at smaller separations and photon noise at larger separations (see Fig. 3, green line). The noise in the total intensity $\delta I$ is dominated by speckles at all separations (see Fig. 3, black line). The small-scale noise introduced by the instrument polarisation is not significant as long as $\delta Q \gg p_{\text {tel }} \cdot \delta I$. In our data, this condition is usually satisfied, since $p_{\text {tel }}$ is on the order of $10^{-3}$ while the noise ratio $\delta I / \delta Q$ is only on the order of 10 . Even though the improvement in point-source contrast is small, we still do the subtraction of the instrument polarisation because we want to reach the best possible contrast and remove as many instrumental effects as possible. 

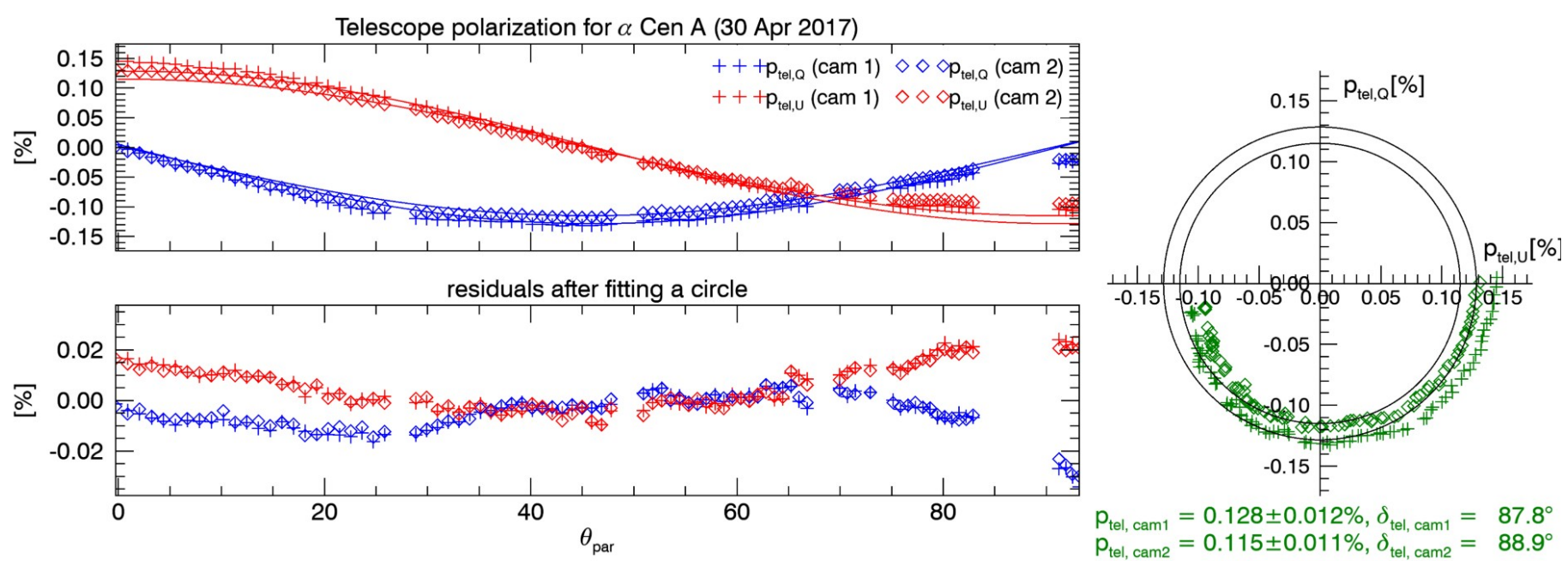

Fig. A.1. Median telescope polarisation for $\alpha$ Cen A from 2017 in N_R-band measured for both Stokes parameters $-Q$ and $U-$ as a function of the telescope parallactic angle $\theta_{\text {par }}$. The plots show the measurements for camera $1(+)$ and camera $2(\diamond)$. The measurements have been fit with a model function (solid lines) resulting in a measurement of the telescope polarisation amplitude $p_{\text {tel }}$ and phase $\delta_{\text {tel }}$ for both cameras.

For the considerations in this section we always assumed that the astrophysical polarisation of the target $Q$ and $U$ are zero when averaged over a large portion of the image. In Appendix B we investigate in more detail what happens to a non-zero astrophysical polarisation signal of a faint companion if we apply the instrumental polarisation correction as described above. We show that the process of subtracting the instrument polarisation as described above has an insignificant effect on the signal of a faint point source potentially present in the data.

\section{A.3. Differential polarimetric beam shift}

The even and odd rows of the detectors in ZIMPOL measure the two opposite linear polarisation states $I_{\|}$and $I_{\perp}$ simultaneously and on the same detector pixels. This would in principle allow for a perfect speckle suppression in the final $Q$ and $U$ polarisation frames. However, it was noticed that $I_{\|}$and $I_{\perp}$ are not perfectly aligned on the detector even though they go through the same optical path in the instrument. This unexpectedly large differential polarisation beam shift of up to 0.3 pixels $(\approx 1$ mas $)$ is caused mostly by reflections on inclined mirrors. The effect is for ZIMPOL described in Schmid et al. (2018) and one example is shown in Fig. A.3. For high-contrast applications the beam shift must be determined and corrected, otherwise the speckles will not cancel sufficiently in the PDI process and a pattern of positive and negative speckle residuals remains.

However, the beam shift, and possibly other differential aberration effects, cannot be corrected perfectly. One reason for this is the wavelength dependence of the beam shift. For broadband observations, this produces radially elongated speckles, for which the innermost (shortest wavelength) part suffers a different beam shift than the outermost (longest wavelength) part. Tests have shown that the beam shift is similar in $R$ and $I$-band, but it can be significantly different in $V$-band. Thus, the wavelength dependence is mainly a problem for observations with the VBB filter because it spans a large portion of the visual spectrum. Observations with any of the $R$ - and $I$-band filters should not suffer as much. Despite this, it is important to apply a "mean" beam-shift correction for any filter, because it can improve the high-contrast performance significantly.

Currently, there exists no comprehensive beam-shift model for the SPHERE/ZIMPOL instrument. Therefore, the first step is a beam-shift measurement, preferentially based on the science data that need to be corrected. This can be achieved with one of the following methods:

- For data taken with the semi-transparent coronagraph CLCMT-WF or without coronagraph, the beam shift can often be measured in individual images as the offset of the PSF peak between even- and odd-row (or $I_{\perp}$ and $I_{\|}$) frames. This is only possible if the PSF peak is not saturated and well defined so that the relative PSF-offsets can be determined with high precision. The observing conditions influence the measuring precision and a careful selection of good beamshift data is essential. For PSF peaks observed through the semi-transparent coronagraph, the determinations are difficult if the peak is close to the "edge" of the coronagraphic flux minimum.

- In some cases, it is easier to derive the beam shift by crosscorrelating the speckle pattern or numerically solving for the differential offset of $I_{\|}$and $I_{\perp}$ that minimizes the residuals in $Q^{Z}=I_{\perp}-I_{\|}$. This method often works well because the beam shift is identical for the whole FOV as shown in Fig. A.3. The minimization works better for shorter exposures and narrow filters because the individual speckles are more numerous and better defined. In long exposures the atmospheric turbulences smooth short-lived speckles and broadband observations extend the shape of the speckles strongly in the radial direction, reducing their signal with respect to the background.

Both methods allow the determination of the beam shift with a precision of order 0.01 pixels $(\approx 0.04$ mas $)$ in a single exposure for data with good observing conditions. For a proper beam-shift correction, the high precision is necessary because the effect is only of the order of 0.1 pixels, but the difference of the correction is noticeable even in a single exposure. Figure A. 3 shows a short exposure of $\alpha$ Cen A before and after applying the beam-shift correction. Most of the residual speckle pattern can be suppressed, significantly improving the point-source contrast limit in the speckle-dominated region. In the combined final image the effect of the beam shift cannot only be seen as additional speckles on a small scale but also as a disturbing feature on a larger scale. This is because the whole speckle halo of the stellar PSF is beam shifted. In our high-contrast images this artificially produces negatively and positively polarised large-scale 

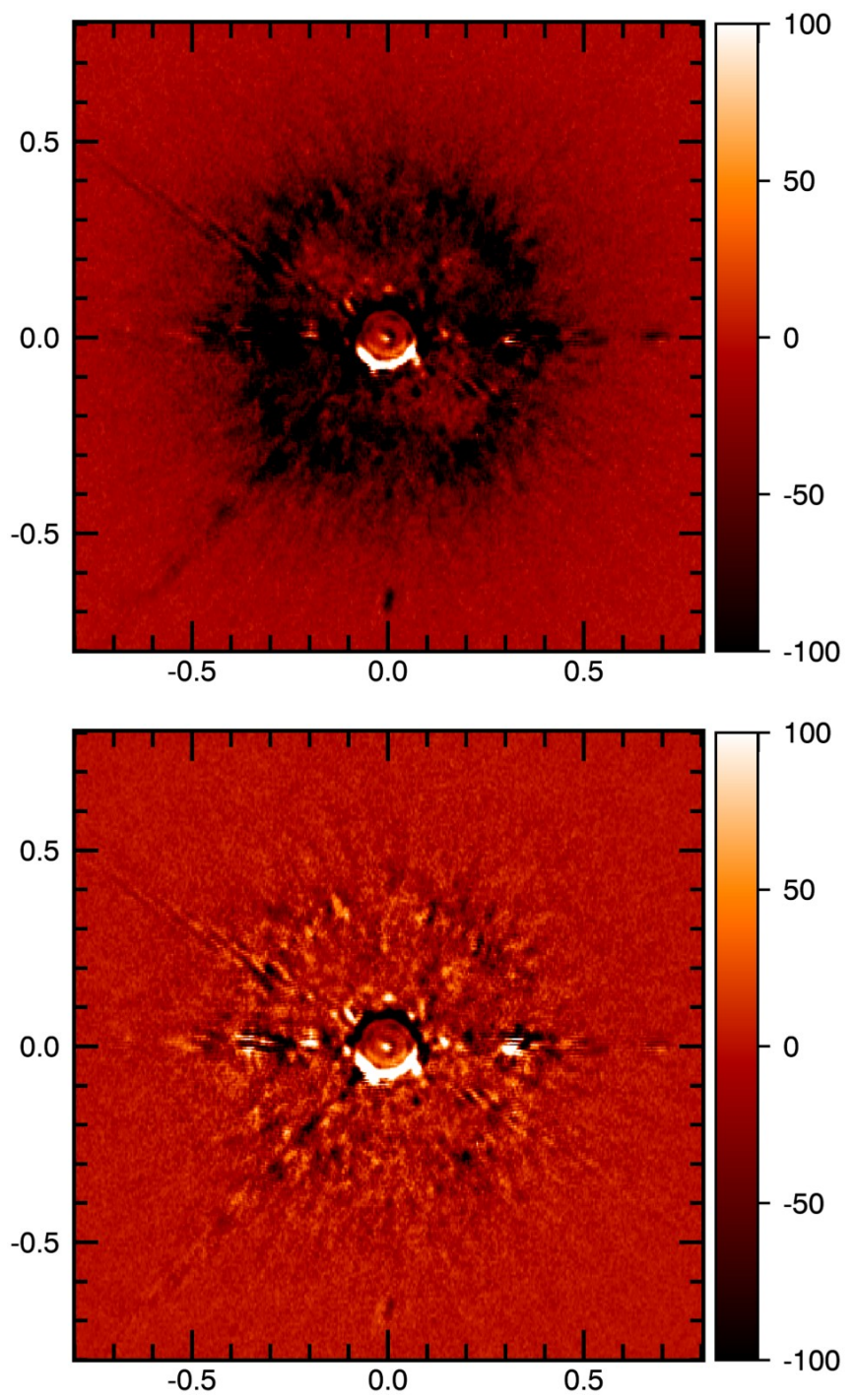

Fig. A.2. Comparison of a coronagraphic exposure before (top) and after (bottom) the subtraction of the telescope polarisation $p_{\text {tel }}$. The images show the polarised intensity $Q$ of a combined pair of one single zero-phase and one $\pi$-phase $(2 \times 1.2 \mathrm{~s})$ exposure of $\alpha$ Cen $\mathrm{A}$ in the N_R filter. The axes are in arc seconds and the colour scale is in ADU.

features that can limit the sensitivity for real large-scale polarised signals.

Unfortunately, the beam-shift correction also introduces some new systematic noise residuals. All intensity features originating from components located downstream of the inclined mirrors - namely the M3 mirror, the pupil tip-tilt mirror, and image de-rotator mirrors - are not subject to the beam-shift effect. Applying the beam-shift correction to the polarimetric data as described above will introduce spurious residual patterns in the "corrected" polarimetric image. This concerns the intensity edges of the attenuating focal plane mask of the coronagraph, or intensity patterns from the dead actuators of the deformable mirror, as well as bad pixels, charge traps, and dust on the micro-lens array of the ZIMPOL detector (Schmid et al. 2018). An example in Fig. A.3 is the black and white pattern visible at the edge of the coronagraph because of the applied beam-shift correction in the vertical direction. This effect increases the effective IWA of the result and cannot be corrected. The intensity patterns from dust on the micro-lens array can be efficiently removed by flat-fielding, and the pixel
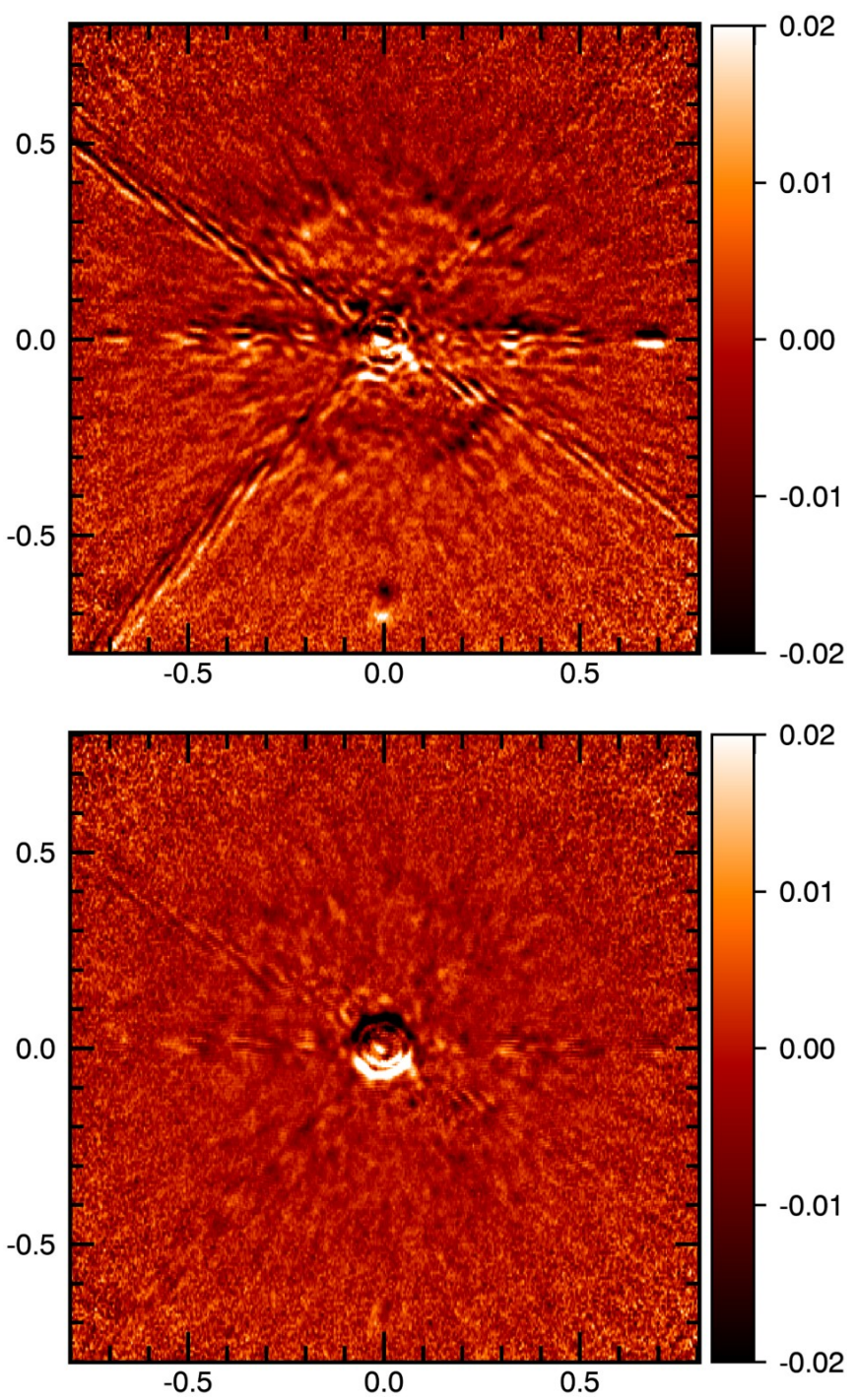

Fig. A.3. Comparison of a coronagraphic exposure before (top) and after (bottom) the beam-shift correction. The images show the fractional polarisation $Q / I$ of a single zero-phase and $\pi$-phase combined $2 \times 1.2 \mathrm{~s}$ exposure of $\alpha$ Cen A in the N_R filter. The axes are in arc seconds and the colour scale is dimensionless.

scale effects can be strongly reduced by masking, bad-pixel cleaning, dithering, or angular differential imaging.

The beam shift changes continuously with the telescope pointing direction and depends on both the parallactic angle $\theta_{\mathrm{par}}$ and altitude angle $\theta_{\text {alt }}$. Therefore, it is advantageous for long observations if the beam shift can be accurately measured from the science data themselves without requiring any additional overheads. However, for coronagraphic data or saturated data, the beam-shift measurement may fail and therefore we regularly take non-coronagraphic PSF measurements.

\section{Appendix B: Subtraction of instrument polarisation}

By measuring the residual polarisation in our data for different parallactic angles $\theta_{\text {par }}$ (see Sect. A.2) we determined that all targets of our survey are only weakly polarised $\left(p_{\text {st }} \lesssim 10^{-4}\right)$. At this low level of polarisation we can no longer distinguish between the intrinsic polarisation of the target and second-order 
instrumental polarisation effects. We have also shown that the residual telescope polarisation $p_{\text {tel }}$ is of order $10^{-3}$. In Sect. A.2 we explain how this polarisation offset can be measured and removed from the data. This is a common way of removing the instrument polarisation and does not affect any polarised signals in the vicinity of the star as long as the stellar PSF is only polarised due to instrumental or interstellar polarisation because both processes only add a constant fractional polarisation offset to all sources in the FOV. In the following section we investigate the effect of the instrumental polarisation correction on a polarised point source if the star itself exhibits an intrinsic polarisation.

We analyse this problem with a model for the observed Stokes $I_{\mathrm{obs}}(x, y)$ and $Q_{\mathrm{obs}}(x, y)$ signal, with $(x, y)$ being the image coordinates (for $U_{\mathrm{obs}}(x, y)$ the analysis is equivalent). In our model the observed intensity distribution $I_{\mathrm{obs}}(x, y)$ consists of contributions from the PSF of the star itself $I_{\mathrm{PSF}, \mathrm{st}}(x, y)$ and an offset PSF from the planet $I_{\mathrm{PSF}, \mathrm{pl}}(x, y)$, scaled with the flux contrast $C_{\text {flux }}(\alpha)$. The intensities $I_{\mathrm{PSF}, \mathrm{st}}(x, y)$ and $I_{\mathrm{PSF}, \mathrm{pl}}(x, y)$ are offset from each other but are otherwise identical. The polarised intensity distribution $Q_{\mathrm{obs}}(x, y)$ consists of the intrinsic polarisation of the star $p_{\mathrm{st}, O} I_{\mathrm{PSF}, \mathrm{st}}(x, y)$, the polarised signal from the planet $C_{\mathrm{pol}, Q}(\alpha) I_{\mathrm{PSF}, \mathrm{pl}}(x, y)$, and a term that describes the polarisation offset due to instrumental and/or interstellar polarisation $p_{\text {tel }, Q}\left(I_{\mathrm{PSF}, \mathrm{st}}(x, y)+C_{\text {flux }}(\alpha) I_{\mathrm{PSF}, \mathrm{pl}}(x, y)\right)$. For the sake of readability, we omit the dependences on scattering angle $\alpha$ and image coordinates $(x, y)$ during the derivations.

$I_{\mathrm{obs}}=I_{\mathrm{PSF}, \mathrm{st}}+C_{\mathrm{flux}} I_{\mathrm{PSF}, \mathrm{pl}}$

$Q_{\mathrm{obs}}=p_{\mathrm{st}, Q} I_{\mathrm{PSF}, \mathrm{st}}+C_{\mathrm{pol}, Q} I_{\mathrm{PSF}, \mathrm{pl}}+p_{\mathrm{tel}, Q}\left(I_{\mathrm{PSF}, \mathrm{st}}+C_{\mathrm{flux}} I_{\mathrm{PSF}, \mathrm{pl}}\right)$.

From Eq. (B.1) we want to extract the signal of the polarised planet with all instrumental and (inter-)stellar contributions removed. The corrected polarised intensity $Q_{\mathrm{obs}}^{\prime}(x, y)$ is therefore

$Q_{\mathrm{obs}}^{\prime}=C_{\mathrm{pol}, Q} I_{\mathrm{PSF}, \mathrm{pl}}=p_{\mathrm{pl}, Q} C_{\mathrm{flux}} I_{\mathrm{PSF}, \mathrm{pl}}$.

The contrasts $C_{\text {flux }}(\alpha)$ and $C_{\mathrm{pol}, Q}(\alpha)$ are linked by the fractional polarisation of the planet $p_{\mathrm{pl}, Q}(\alpha)=C_{\mathrm{pol}, Q}(\alpha) / C_{\mathrm{flux}}(\alpha)$. Fractional polarisations have an additional subscript $Q$ that can be either negative or positive because they are entries of the twodimensional vector for the linear polarisation $p=\left(p_{Q}, p_{U}\right)$ in the Stokes $Q-U$ plane. All of the derivations here are equivalent for the Stokes $U$ measurements.

Using this model, the flux-weighted fractional polarisation in a large aperture $\left\langle Q_{\mathrm{obs}}\right\rangle /\left\langle I_{\mathrm{obs}}\right\rangle$ is given by

$\frac{\left\langle Q_{\mathrm{obs}}\right\rangle}{\left\langle I_{\mathrm{obs}}\right\rangle}=\frac{\left\langle p_{\mathrm{st}, Q} I_{\mathrm{PSF}, \mathrm{st}}+C_{\mathrm{pol}, Q} I_{\mathrm{PSF}, \mathrm{pl}}\right\rangle}{\left\langle I_{\mathrm{PSF}, \mathrm{st}}+C_{\mathrm{flux}} I_{\mathrm{PSF}, \mathrm{pl}}\right\rangle}+p_{\mathrm{tel}, Q}$.

We usually do not know the exact intrinsic polarisation of the star, except that it is small, and contributions from the planet, except that they are very faint. Therefore, we correct the telescope polarisation by simply assuming that the two contributions can be neglected and subtract the scaled intensity $I_{\text {obs }}$ as follows:

$Q_{\text {obs,norm }}=Q_{\text {obs }}-\frac{\left\langle Q_{\text {obs }}\right\rangle}{\left\langle I_{\text {obs }}\right\rangle} I_{\text {obs }}$.

We then combine Eqs. (B.3) and (B.4) and notice that all terms with $p_{\text {tel }, Q}$ have successfully vanished:

$$
\begin{aligned}
Q_{\mathrm{obs}, \mathrm{norm}}= & p_{\mathrm{st}, Q} I_{\mathrm{PSF}, \mathrm{st}}+C_{\mathrm{pol}, Q} I_{\mathrm{PSF}, \mathrm{pl}} \\
& -\frac{p_{\mathrm{st}, Q}\left\langle I_{\mathrm{PSF}, \mathrm{st}}\right\rangle+C_{\mathrm{pol}, Q}\left\langle I_{\mathrm{PSF}, \mathrm{pl}}\right\rangle}{\left\langle I_{\mathrm{PSF}, \mathrm{st}}\right\rangle+C_{\mathrm{flux}}\left\langle I_{\mathrm{PSF}, \mathrm{pl}}\right\rangle}\left(I_{\mathrm{PSF}, \mathrm{st}}+C_{\mathrm{flux}} I_{\mathrm{PSF}, \mathrm{pl}}\right)^{.}
\end{aligned}
$$

We define $f_{\mathrm{pl}, \mathrm{st}}=\left\langle I_{\mathrm{PSF}, \mathrm{pl}}\right\rangle /\left\langle I_{\mathrm{PSF}, \mathrm{st}}\right\rangle$ then simplify the result by factoring out the stellar and planetary PSFs:

$$
\begin{aligned}
Q_{\mathrm{obs}, \text { norm }}= & -C_{\mathrm{flux}} I_{\mathrm{PSF}, \mathrm{st}} f_{\mathrm{pl}, \mathrm{st}} \frac{p_{\mathrm{pl}, Q}-p_{\mathrm{st}, Q}}{1+C_{\mathrm{flux}} f_{\mathrm{pl}, \mathrm{st}}} \\
& +C_{\text {flux }} I_{\mathrm{PSF}, \mathrm{pl}} \frac{p_{\mathrm{pl}, Q}-p_{\mathrm{st}, Q}}{1+C_{\mathrm{flux}} f_{\mathrm{pl}, \mathrm{st}}} .
\end{aligned}
$$

Now we can estimate the order of magnitude of the different terms for our particular case:

- $f_{\mathrm{pl}, \mathrm{st}}$ corresponds to the total number of counts in the planetary PSF divided by the number of counts in the stellar PSF measured in the ring of pixels around the star that was used to calculate $\langle\ldots\rangle$. We determined that the value of this parameter for the $\alpha$ Cen A data is $\lesssim 3$ if the planetary PSF is inside the ring and it is always smaller than one if the planetary PSF is outside the ring.

- The flux contrast of a reflecting planet $C_{\text {flux }}$ is expected to be of order $10^{-7}$ or smaller.

Considering the simplifications above, we can approximate $(1+$ $\left.C_{\text {flux }} f_{\mathrm{pl}, \mathrm{st}}\right) \approx 1$ and simplify Eq. (B.6) to:

$Q_{\mathrm{obs}, \text { norm }} \approx C_{\mathrm{flux}}\left(p_{\mathrm{pl}, Q}-p_{\mathrm{st}, Q}\right) I_{\mathrm{PSF}, \mathrm{pl}}\left(1-f_{\mathrm{pl}, \mathrm{s}} \frac{I_{\mathrm{PSF}, \mathrm{st}}}{I_{\mathrm{PSF}, \mathrm{pl}}}\right)$.

Equation (B.7) still shows two additional terms compared to the desired result in Eq. (B.2). The expression in the right hand bracket in Eq. (B.7) mainly depends on the separation of the stellar and planetary PSFs because for this term to have a small contribution to $Q_{\mathrm{obs}, \mathrm{norm}}$ the value of $I_{\mathrm{PSF}, \mathrm{st}}(x, y) / I_{\mathrm{PSF}, \mathrm{pl}}(x, y)$ needs to be small at the $(x, y)$-position where the planet PSF peaks. The PSFs for the $\alpha$ Cen A observation in Fig. 6 show that for any separation larger than the IWA of about $0.15^{\prime \prime}$ this ratio is smaller than $\sim 10^{-3}$ and the whole expression in the right hand bracket does not reduce the $Q$ signal of a planet by more than $0.3 \%$. If we neglect this small correction factor, we finally arrive at the following expression for the telescope polarisation corrected (or normalized) $Q$ image,

$Q_{\text {obs,norm }}(x, y) \approx C_{\text {flux }}\left(p_{\mathrm{pl}, Q}(\alpha)-p_{\mathrm{st}, Q}\right) I_{\mathrm{PSF}, \mathrm{pl}}(x, y)$,

where we re-introduced the correct dependences of the parameters on scattering angle $\alpha$ and image coordinates $(x, y)$. The result means that for a high-contrast point source, our process of removing the instrument polarisation modifies the fractional polarisation of the planet $p_{\mathrm{pl}, Q}(\alpha)$ with the intrinsic fractional polarisation of the star $p_{\mathrm{st}, Q}$. However, given that we have shown (see Appendix A.2) that the polarisation of the star $p_{\text {st }}$ is $\lesssim 2 \times 10^{-4}$ for all our targets, we expect the change of the observed polarisation of the planet to be $\lesssim 0.002 \%$. This is insignificant compared to the expected $p_{\mathrm{pl}, Q}(\alpha) \approx 10 \%$ of a reflecting planet, and therefore we conclude that our process of removing the instrument polarisation does not significantly effect the polarised signal of a planet in our specific case.

\section{Appendix C: Results and discussion for the additional targets}

\section{C.1. $\alpha$ Centauri B}

In Fig. C.1 we show the deepest contrast limits for $\alpha$ Cen B, derived from a dataset with a total $t_{\exp }$ of $206.8 \mathrm{~min}$ in the VBB filter during a half-night with excellent observing conditions (see Table 1). $\alpha$ Cen B is about four times fainter than 


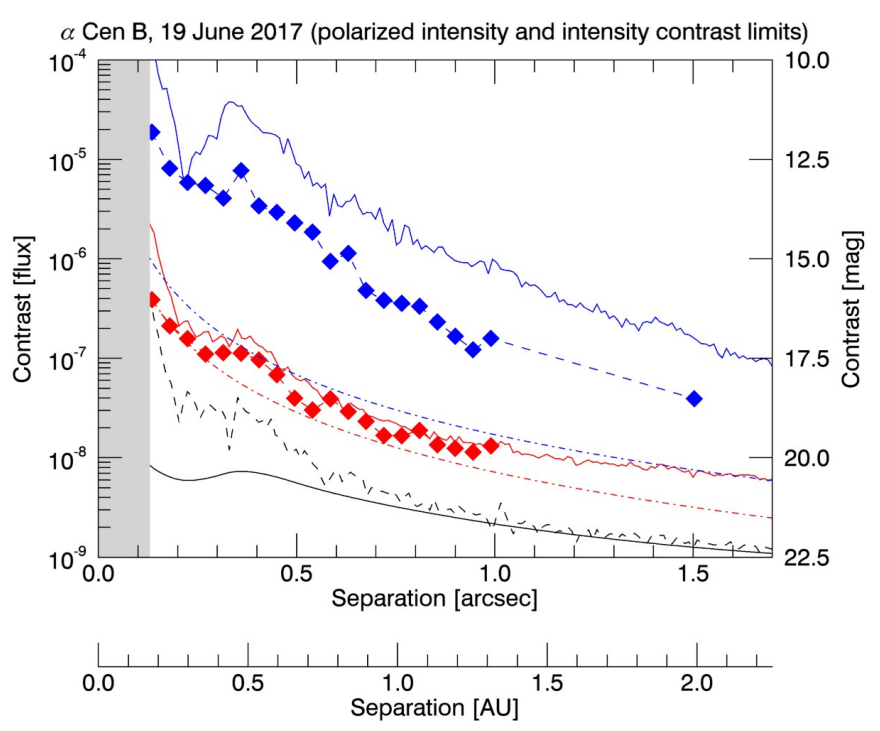

Fig. C.1. Radial contrast limits as a function of separation for the deepest ZIMPOL high-contrast dataset of $\alpha$ Cen B in the VBB filter. The plot shows the $5 \sigma$ limits for the intensity and polarised intensity from the same observation as well as the $1 \sigma$ photon noise limit for the polarised intensity. The meaning of the colours and symbols is the same as explained in Fig. 6.

the A component but using the VBB filter instead of the N_R (or N_I) filter compensates for this, resulting in similar count rates and contrast limits to those obtained for component $\mathrm{A}$. The raw polarimetric contrast can be improved considerably with the use of PCA-ADI, even surpassing the limits for $\alpha$ Cen $\mathrm{A}$ for some separations. Because of the excellent observing conditions, despite using the shortest possible DIT of $1.1 \mathrm{~s}$, some frames have saturated pixels just at the edge of the coronagraph located within the effective IWA of the data (highlighted by the grey bar in the contrast curve plots).

From a stability point of view, there is no reason why it should not be possible for $\alpha$ Cen B to harbour a Jupiter-sized planet, just like for $\alpha$ Cen A. However, for the B component of the system, the RV limits are much more stringent than for the A component. The radial velocity limits for $\alpha$ Cen B from Zhao (2018) exclude planets with $M \sin (i)>8.4 M_{\text {Earth }}$ for the classically defined habitable zone from about 0.7-1.3 AU, and even more stringent limits for smaller separations.

For $\alpha$ Cen A we have discussed some reasons why the RV limits would still allow a giant planet to be in orbit around this star and that our deep contrast limits could allow us to observe such a giant planet. Due to the stringent RV limits however, the arguments cannot be applied to $\alpha$ Cen B. Orbital inclinations with $\sin (i) \lesssim 0.5$ are unlikely due to stability arguments. The resulting optimistic upper limit for the mass of a potential companion of $\sim 20 M_{\text {Earth }}$, comparable to the mass of Neptune or Uranus, makes it unlikely that $\alpha$ Cen B could harbour a planet large enough to be detectable with the limits presented in Fig. C.1 for a single half-night. The possibility of detecting a low-mass planet around $\alpha$ Cen B with ZIMPOL was discussed by Milli et al. (2013) in light of the former exoplanet candidate $\alpha \mathrm{Cen} \mathrm{Bb}$. These latter authors concluded that a detection of the reflected light should be possible. However, the study focused on very close separations and the use of a four-quadrant phase-mask coronagraph (not commissioned for ZIMPOL). Such small separations are mostly inaccessible with the Lyot coronagraph used in our survey.

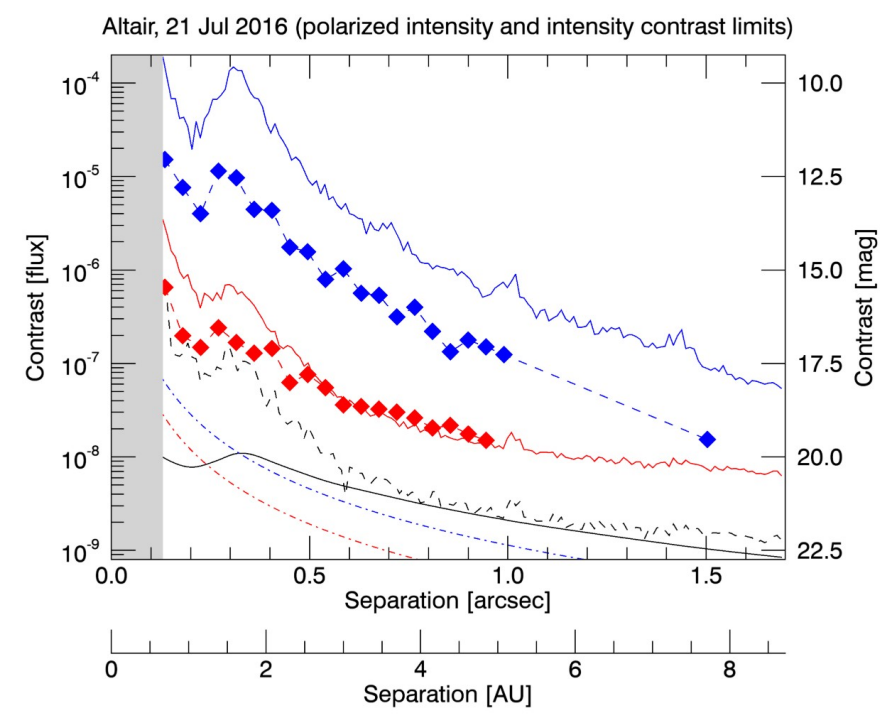

Fig. C.2. Radial contrast limits as a function of separation for the deepest ZIMPOL high-contrast dataset of Altair in the R_PRIM filter. The plot shows the $5 \sigma$ limits for the intensity and polarised intensity from the same observation as well as the $1 \sigma$ photon noise limit for the polarised intensity. The meaning of the colours and symbols is the same as explained in Fig. 6.

A previous search around $\alpha$ Cen $\mathrm{B}$ was again done by Schroeder et al. (2000) with HST. The contrast limits are comparable to $\alpha$ Cen A; between $0.5^{\prime \prime}$ and $1.5^{\prime \prime}$ the limits are between 7.5 and 8.5 mag at a wavelength of $\sim 1.02 \mu \mathrm{m}$. Our contrast limits (see Fig. C.1 and summary in Table 2) push these limits for the $R+I$-band by a large amount.

\section{C.2. Altair}

In Fig. C. 2 we show the deepest contrast limits for Altair derived from a dataset with a total $t_{\exp }$ of $151.3 \mathrm{~min}$ in the R_PRIM filter. The filter is $\sim 2.6$ times broader than the narrow $R$-band filter used for $\alpha$ Cen A, but Altair is fainter by about the same factor. This results in similar numbers for the captured photons per second and similar contrast limits. The main difference is the larger distance, and therefore the lower expected signal for given planet parameters and angular separation, as shown for our model planet in Fig. C.2.

Altair is an active star and a fast rotator, which makes it difficult to use the RV method to determine precise upper mass limits for possible companions. For example, the survey from Lagrange et al. (2009) shows that the RV limits for planets around a fast rotating early type star like Altair allow only the detection of high-mass and short-period exoplanets. Therefore, a direct imaging search is competitive and complementary with respect to the RV studies.

The deepest contrast limits for Altair were derived in the HST survey of Schroeder et al. (2000) conducted with the HST who achieved about 7.5-8.5 mag for a wavelength of $\sim 1.02 \mu \mathrm{m}$ and separations of $0.5^{\prime \prime}-1.5^{\prime \prime}$. Our limits in intensity are about 12.8-17.7 mag and in polarised intensity are 17.9-20.3 mag but with an effective IWA of only $\sim 0.13^{\prime \prime}$. The limits are deep in terms of contrast; however, with 5 pc Altair is the most distant object in our survey. As a result, the polarimetric contrast of the reflected light from a Jupiter-sized planet with our model atmosphere would be lower than $10^{-8}$ for all separations larger than $0.22^{\prime \prime}$. Reaching such contrast levels is not possible with only a few hours of observation. 

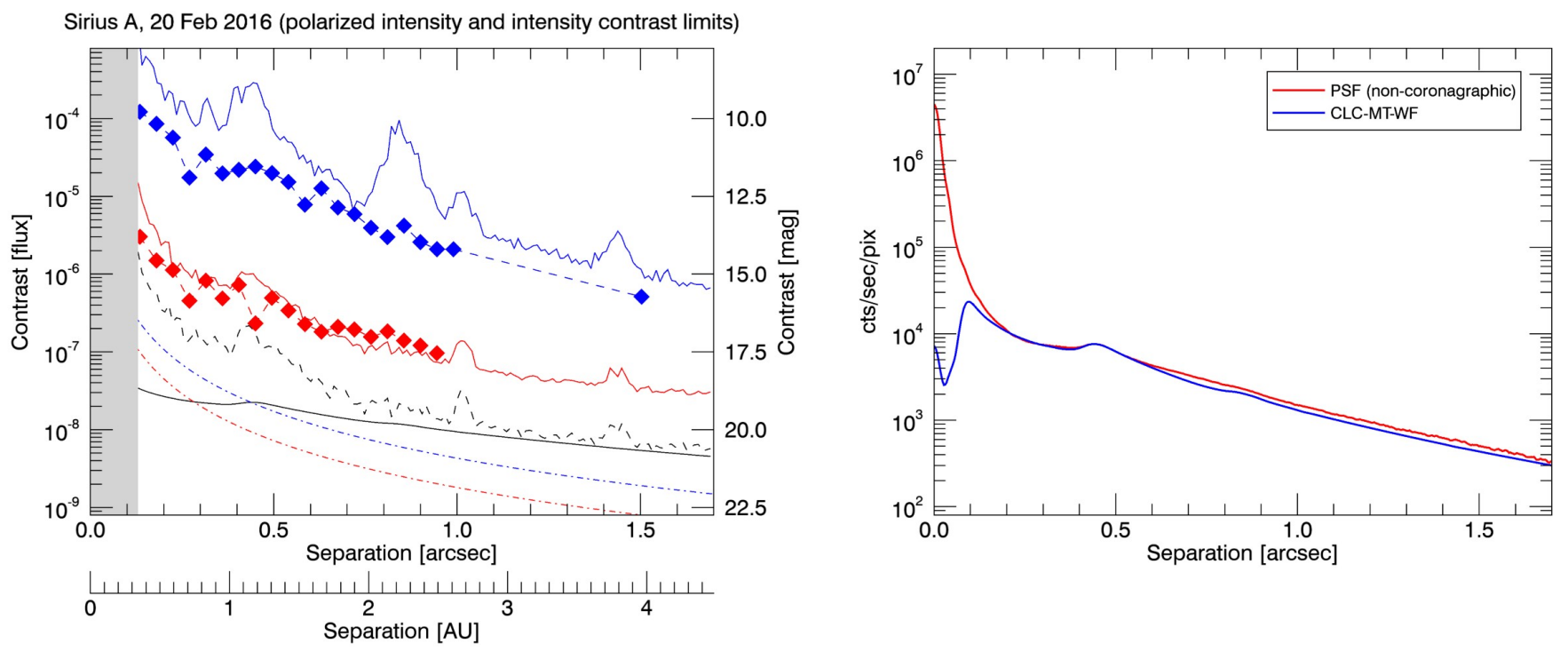

Fig. C.3. Left: radial contrast limits as a function of separation for the deepest ZIMPOL high-contrast dataset of Sirius A in the N_I filter. The plot shows the $5 \sigma$ limits for the intensity and polarised intensity from the same observation as well as the $1 \sigma$ photon noise limit for the polarised intensity. The meaning of the colours and symbols is the same as explained in Fig. 6. Right: coronagraphic PSF compared to the non-coronagraphic PSF of Sirius A in the N_I filter.

\section{C.3. Sirius $A$}

In Fig. C. 3 we show the deepest contrast limits for Sirius A based on a total $t_{\text {exp }}$ of $175.2 \mathrm{~min}$. The N_I filter was used to avoid saturation of this very bright blue star in coronagraphic mode with the shortest possible DIT. Unfortunately, the atmospheric conditions for these observations were poor (see Table 1), resulting in a degradation of the resolution and the contrast, which makes it more difficult to perform some of the data-reduction steps (e.g. centring, beam-shift correction, ...). The resulting contrast limits are much worse than what would be possible for such a bright target. The coronagraphic PSF in the right panel in Fig. C.3 clearly shows that the level of the PSF halo is enhanced and the noncoronagraphic PSF peak is significantly lowered when compared to the PSFs from the observation of $\alpha$ Cen A shown in Fig. 5. Both effects have a negative impact on the $\mathrm{S} / \mathrm{N}$ of a point source and the contrast limit of the data.

The radial velocity mass and separation limits for low-mass objects are loose for Sirius A because it is an intermediatemass $\left(\sim 2 M_{\odot}\right)$ A1V star with strong intrinsic RV variation (e.g. Lagrange et al. 2009). The possibility for stable planetary orbits around Sirius A was investigated by Holman \& Wiegert (1999) and Bond et al. (2017), suggesting that stable orbits with periods up to $2.24 \mathrm{yr}$ are possible. This corresponds to a semimajor axis of 2.2 AU or $0.83^{\prime \prime}$ in angular separation. Bond et al. (2017) used precise HST astrometry and could not exclude the presence of a third body in the system with a mass smaller than 15-25 $M_{\text {Jupiter }}$.

There have been attempts to find massive companions to Sirius A in the infrared by Schroeder et al. (2000) using HST and reaching a contrast limit of about 7.5-8.5 mag between $0.5^{\prime \prime}$ and $1.5^{\prime \prime}$ for a wavelength of $\sim 1.02 \mu \mathrm{m}$. Thalmann et al. (2011) used Subaru IRCS and AO188 in the $4.05 \mu \mathrm{m}$ narrowband $\mathrm{Br} \alpha$ filter. At an IWA of $0.7^{\prime \prime}$ they were able to achieve a contrast of about $11 \mathrm{mag}$, and about $14 \mathrm{mag}$ at a separation of $1.5^{\prime \prime}$. The deepest limits were obtained with the IRDIS and IFS instrument of SPHERE/VLT in the near-infrared from 0.95 to $2.3 \mu \mathrm{m}$. (Vigan et al. 2015) using SDI in combination with ADI with an IWA of only $0.2^{\prime \prime}$. These latter authors report contrasts up to $14.3 \mathrm{mag}$ at $0.2^{\prime \prime}$ and $\sim 16.3 \mathrm{mag}$ in the $0.4-1.0^{\prime \prime}$ range. With our combination of PDI and ADI we achieved slightly better contrasts of about $14.7 \mathrm{mag}$ at $0.2^{\prime \prime}$ and $\sim 17.1 \mathrm{mag}$ in the $0.4-1.0^{\prime \prime}$ range in $I$-band $(\lambda=817 \mathrm{~nm})$ with a smaller effective IWA of $\sim 0.13^{\prime \prime}$. However, our observation suffers from poor observing conditions, and contrast limits like for the other bright targets in our survey (e.g. $\alpha$ Cen A/B) should be possible for Sirius A as well under good seeing conditions. This would improve our polarised intensity contrast limits by about 3 mag at all separations.

Our contrast limits are relatively far away from detecting the Jupiter-sized reference model planet when comparing to $\alpha$ Cen A/B. This is partially due to the poor observing conditions and partially due to the distance of $2.64 \mathrm{pc}$ which is about twice as far as $\alpha$ Cen. Because the contrast of a companion scales for a given angular separation like $L[p c]^{-2}$ with distance $L$, the reflected light contrast for a reference planet at the same angular separation to its host star is four times more demanding for Sirius A compared to $\alpha$ Cen. For bright stars $<1.5^{m}$, the contrast efficiency of ZIMPOL is limited by the frame rate, or the ability to collect as many photons as possible without saturating the coronagraphic images. Therefore, the $C \propto L^{-2}$ is increasing the required $t_{\exp }$ for a detection of a planet in reflected light around Sirius A by a factor of 16 when compared to $\alpha$ Cen A/B. However, for smaller separations $\lesssim 0.3^{\prime \prime}$ the reflected light contrast of a possible Jupiter-sized planet around Sirius A increases to values above $2 \times 10^{-8}$ which could be in reach for ZIMPOL within a few consecutive observing nights, assuming a $t_{\exp }^{-1 / 2}$ noise scaling.

\section{C.4. $\tau$ Ceti}

In Fig. C. 4 we show the deepest contrast limits for a half-night of observing $\tau$ Ceti during a time with excellent observing conditions (see Table 1) derived from a total $t_{\exp }$ of $168 \mathrm{~min}$ in the R_PRIM filter. We used a long $t_{\text {DIT }}=14 \mathrm{~s}$ per exposure to ensure that the contrast is photon noise limited in the whole ZIMPOL FOV. One problem with long exposures in P1 mode is rotational smearing. The field rotation can be quite fast because $\tau$ Ceti passes close to the zenith. We could have used the broader VBB filter for this observation to maximize the number of 


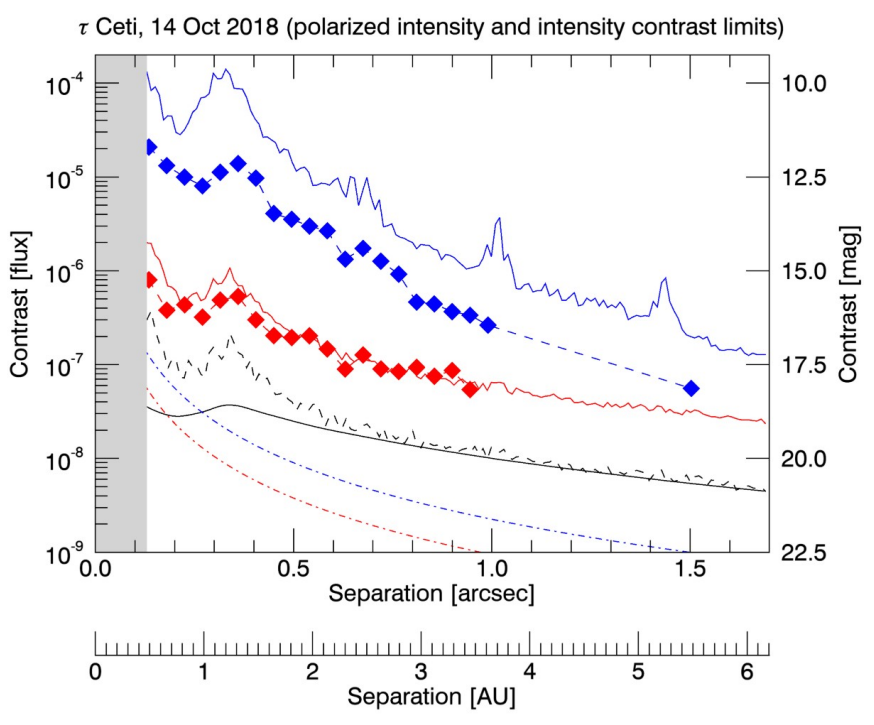

Fig. C.4. Radial contrast limits as a function of separation for the deepest ZIMPOL high-contrast dataset of $\tau$ Ceti in the R_PRIM filter. The plot shows the $5 \sigma$ limits for the intensity and polarised intensity from the same observation as well as the $1 \sigma$ photon noise limit for the polarised intensity. The meaning of the colours and symbols is the same as in Fig. 6, except that we only subtracted 10 PCs during the PCA-ADI step for the polarised intensity. For this dataset the resulting contrast limits were significantly better with only 10 instead of the 20 PCs that we used for all other datasets.

collected photons, however we selected R_PRIM because some of the instrumental effects (e.g. instrument polarisation, beam shift) can be corrected more accurately in the data reduction for the narrower filters because of strongly wavelength-dependent effects which increase with filter width. This strategy seems to be beneficial for the planet search at small separations of $<0.4^{\prime \prime}$ where the speckle noise dominates, while it is less favourable at larger separations in the photon-noise-limited region. The resulting contrast limits for one half-night for $\tau$ Ceti are not as deep as for most other targets in our survey because the resulting photon counts are about ten times lower than for our brighter targets.

The presence of RV planets around $\tau$ Ceti has been proposed by Tuomi et al. (2013) and Feng et al. (2017). However, the measured signals indicate masses $M \sin (i) \lesssim 6.6 M_{\text {Earth }}$ and such planets would be too faint to be observable in our data. Highmass planets $>3 M_{\text {Jupiter }}$ are excluded by a separate study based on Gaia and HipPARCOS astrometry for the separation range 3-30 AU or $\approx 1-10^{\prime \prime}$ (Kervella et al. 2019).

Deep direct imaging contrast limits for $\tau$ Ceti are given by Schroeder et al. (2000) who achieved between 9.0 and 11.5 mag in the separation range from $0.5^{\prime \prime}$ to $1.5^{\prime \prime}$ with HST at a wavelength of $\sim 1.02 \mu \mathrm{m}$. At longer wavelengths, Boehle et al. (2019) report a limiting contrast of about 11.0-12.0 mag in $L$-band with NACO at the VLT in the separation range from $1.0^{\prime \prime}$ to $1.5^{\prime \prime}$. With one excellent night we were able to achieve contrast limits in intensity of about 12.3-16.9 mag and limits in polarised intensity of 16.7-18.8 mag for the separation range from $0.5^{\prime \prime}$ to $1.5^{\prime \prime}$ but with an IWA down to $\sim 0.13^{\prime \prime}$. The contrast limits are deep but - as shown in Fig. C. 4 - they are still far above what we calculate for our model Jupiter-sized planet, both in intensity and polarised intensity. This is because with $3.65 \mathrm{pc}, \tau$ Ceti is one of the more distant targets and with $m_{\mathrm{R}}=2.9$ also one of the fainter targets of our survey. As a result, the polarimetric contrast of the reflected light from our Jupiter-sized reference planet

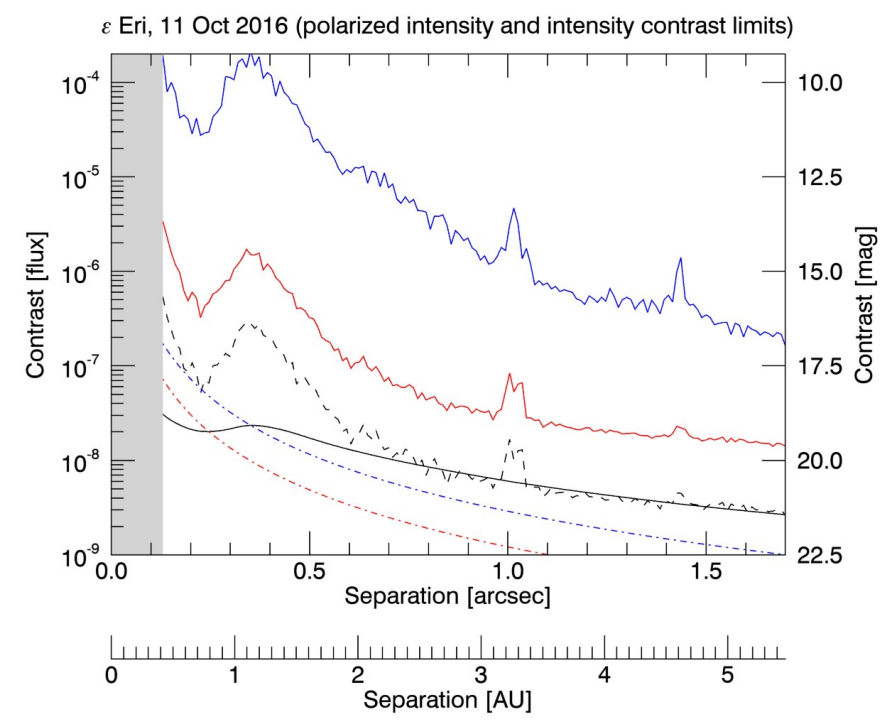

Fig. C.5. Radial contrast limits as a function of separation for the deepest ZIMPOL high-contrast dataset of $\epsilon$ Eri in the VBB filter. The plot shows the $5 \sigma$ limits for the intensity and polarised intensity from the same observation as well as the $1 \sigma$ photon noise limit for the polarised intensity. The meaning of the colours and symbols is the same as in Fig. 6.

model would be lower than $10^{-8}$ for any separations larger than $0.3^{\prime \prime}$.

It is known that $\tau$ Ceti hosts a large debris disk and Lawler et al. (2014) measured with Herschel an inner edge between 1 and $10 \mathrm{AU}$, an outer edge at about $\sim 55 \mathrm{AU}$, and an inclination of $35^{\circ} \pm 10^{\circ}$ from face-on. The total mass of the disk is estimated to be only $\sim 1 M_{\text {Earth }}$ (Greaves et al. 2004) and it is extended over a large range of separations. We did not detect the signal of an extended source around $\tau$ Ceti in our data, and therefore it is either too faint to be seen directly in our data or the inner edge is located outside of our FOV of about $1.7^{\prime \prime}$ or about $6 \mathrm{AU}$ at this distance.

\section{C.5. $\epsilon$ Eridani}

In Fig. C.5 we show the deepest contrast limits of $\epsilon$ Eri for a single half-night with good observing conditions (see Table 1) and a total $t_{\text {exp }}$ of 192 min in the VBB filter. This is the only target observed in the field stabilised P2 polarimetry mode. Without field rotation, we cannot apply ADI to this dataset. The targets $\epsilon$ Eri and $\tau$ Ceti are almost identical in brightness and therefore it is interesting to compare the contrast limits of the non-ADI $\epsilon$ Eri data with the ADI $\tau$ Ceti data and the different filters used. The achieved contrast for $\epsilon$ Eri is significantly deeper at larger separations because of the longer total $t_{\exp }$ and the broader filter, and therefore increased photon counts by a factor of approximately two. At closer separations in the speckle-dominated regime the contrast limits for $\tau$ Ceti are better, despite the smaller number of collected photons. The ADI data of $\tau$ Ceti suffer less quasi-static aberrations because the speckles are averaged and significantly reduced by the PSF subtraction. From this comparison we estimate that field rotation would improve the contrast limits for $\epsilon$ Eri inside the speckle ring by up to a factor of five for the polarisation up to 10 for the intensity, but at the time of the observation the addition of ADI was not yet considered to be an option. 
The deepest direct imaging and radial velocity limits for $\epsilon$ Eri are both presented in Mawet et al. (2019). They also present the strongest evidence so far for the existence of $\epsilon$ Eri b; a giant planet with a mass of $\sim 1.2 M_{\text {Jupiter }}$ for an orbital inclination of $34^{\circ} \pm 2^{\circ}$ when assumed to be coplanar with the outer debris disk. The star is also monitored by Gaia but the measured astrometric trends are not yet precise enough to confirm the planet (Kervella et al. 2019). The planet's separation of $\sim 3.5 \mathrm{AU}$ is well within the FOV of ZIMPOL and in the photon noise dominated regime of the contrast curve (see Fig. C.5). However, we were not able to detect the planet because a Jupiter-sized reference model planet would produce a polarisation contrast below $1.2 \times 10^{-9}(22.3 \mathrm{mag})$ at this orbital separation. Our $5 \sigma$ contrast limit with one half-night of observation at this separation is $2.2 \times 10^{-8}(19.1 \mathrm{mag})$. We were also not able to spot any extended polarised emission from the disk around $\epsilon$ Eri. The well-known part of the disk around $\epsilon$ Eri as seen by Herschel and ALMA is located between 11 and 13 AU (Greaves et al. 2014; Booth et al. 2017), which is outside of our FOV with ZIMPOL.

The deepest high-spatial-resolution imaging limits for the thermal emission of $\epsilon$ Eri $b$ were obtained with VLT/NACO in $L p$-band (Mizuki et al. 2016) and Keck/NIRC2 in $M s$-band (Mawet et al. 2019). The $5 \sigma$ contrast curves presented in Mawet et al. (2019) range from $0.3^{\prime \prime}$ to $1.5^{\prime \prime}$ with contrast limits in the range from $\sim 9.0$ to $11.8 \mathrm{mag}$ and $\sim 10.3$ and $13.5 \mathrm{mag}$ for $L p$-band and $M s$-band, respectively. With our best half-night we were able to achieve contrast limits of about 10.0-16.3 mag in reflected intensity and 15.0-19.6 mag in polarised intensity for the same range of separations with an effective IWA of $\sim 0.13^{\prime \prime}$. Due to the broad passband of the VBB filter the contrast limits for $\epsilon$ Eri are deep at the separation where $\epsilon$ Eri b is expected to be orbiting but this is still a factor of $\sim 20$ or $\sim 3.3$ mag away from the expected signal for planet $b$. 